\title{
A MOLECULAR LINE SCAN IN THE HUBBLE DEEP FIELD NORTH
}

\author{
R. Decarli ${ }^{1}$, F. Walter ${ }^{1}$, C. Carilli ${ }^{2}$, D. Riechers ${ }^{3}$, P. Cox $^{4}$, R. Neri $^{4}$, M. Aravena $^{5,6}$, E. Bell $^{7}$, F. Bertoldi $^{8}$, \\ D. Colombo ${ }^{1}$, E. Da Cunha ${ }^{1}$, E. Daddi ${ }^{9}$, M. Dickinson ${ }^{10}$, D. Downes ${ }^{4}$, R. Ellis ${ }^{11}$, L. Lentati ${ }^{12}$, R. Maiolino ${ }^{12}$, \\ K. M. Menten ${ }^{13}$, H.-W. Rix ${ }^{1}$, M. Sargent ${ }^{9}$, D. Stark ${ }^{14}$, B. Weiner ${ }^{14}$, and A. Weiss ${ }^{13}$ \\ ${ }^{1}$ Max-Planck Institut für Astronomie, Königstuhl 17, D-69117 Heidelberg, Germany; decarli@ mpia.de \\ ${ }^{2}$ NRAO, Pete V. Domenici Array Science Center, P.O. Box O, Socorro, NM 87801, USA \\ ${ }^{3}$ Cornell University, 220 Space Sciences Building, Ithaca, NY 14853, USA \\ ${ }^{4}$ IRAM, 300 rue de la Piscine, F-38406 Saint-Martin d'Hères, France \\ ${ }^{5}$ European Southern Observatory, Alonso de Cordova 3107, Casilla 19001, Vitacura Santiago, Chile \\ ${ }^{6}$ Núcleo de Astronomía, Facultad de Ingeniería, Universidad Diego Portales, Av. Ejército 441, Santiago, Chile \\ ${ }^{7}$ Department of Astronomy, University of Michigan, 500 Church Street, Ann Arbor, MI 48109, USA \\ ${ }^{8}$ Argelander Institute for Astronomy, University of Bonn, Auf dem Hügel 71, D-53121 Bonn, Germany \\ ${ }^{9}$ Laboratoire AIM, CEA/DSM-CNRS-Universite Paris Diderot, Irfu/Service d'Astrophysique, \\ CEA Saclay, Orme des Merisiers, F-91191 Gif-sur-Yvette cedex, France \\ ${ }^{10}$ National Optical Astronomy Observatory, 950 North Cherry Avenue, Tucson, AZ 85719, USA \\ ${ }^{11}$ Astronomy Department, California Institute of Technology, MC105-24, Pasadena, CA 91125, USA \\ ${ }^{12}$ Cavendish Laboratory, University of Cambridge, 19 J. J. Thomson Avenue, Cambridge CB3 OHE, UK \\ ${ }_{13}$ Max-Planck-Institut für Radioastronomie, Auf dem Hügel 69, D-53121 Bonn, Germany \\ ${ }^{14}$ Steward Observatory, University of Arizona, 933 North Cherry Street, Tucson, AZ 85721, USA \\ Received 2013 August 16; accepted 2013 December 19; published 2014 January 30
}

\begin{abstract}
We present a molecular line scan in the Hubble Deep Field North (HDF-N) that covers the entire $3 \mathrm{~mm}$ window (79-115 GHz) using the IRAM Plateau de Bure Interferometer. Our CO redshift coverage spans $z \lesssim 0.45$, $1 \lesssim z \lesssim 1.9$ and all $z \gtrsim 2$. We reach a CO detection limit that is deep enough to detect essentially all $z>1 \mathrm{CO}$ lines reported in the literature so far. We have developed and applied different line-searching algorithms, resulting in the discovery of 17 line candidates. We estimate that the rate of false positive line detections is $\sim 2 / 17$. We identify optical/NIR counterparts from the deep ancillary database of the HDF-N for seven of these candidates and investigate their available spectral energy distributions. Two secure $\mathrm{CO}$ detections in our scan are identified with star-forming galaxies at $z=1.784$ and at $z=2.047$. These galaxies have colors consistent with the " $B z K$ " color selection and they show relatively bright $\mathrm{CO}$ emission compared with galaxies of similar dust continuum luminosity. We also detect two spectral lines in the submillimeter galaxy HDF 850.1 at $z=5.183$. We consider an additional nine line candidates as high quality. Our observations also provide a deep $3 \mathrm{~mm}$ continuum map ( $1 \sigma$ noise level $=8.6 \mu \mathrm{Jy}_{\text {beam }}{ }^{-1}$ ). Via a stacking approach, we find that optical/MIR bright galaxies contribute only to $<50 \%$ of the star formation rate density at $1<z<3$, unless high dust temperatures are invoked. The present study represents a first, fundamental step toward an unbiased census of molecular gas in "normal" galaxies at high- $z$, a crucial goal of extragalactic astronomy in the ALMA era.
\end{abstract}

Key words: galaxies: evolution - galaxies: ISM - galaxies: star formation - galaxies: statistics - instrumentation: interferometers - submillimeter: galaxies

Online-only material: color figures

\section{INTRODUCTION}

Studies of the molecular medium in distant galaxies provide key diagnostics about the evolutionary state of galaxies in the high-redshift universe. To date, $\sim 200$ high-redshift galaxies have been detected in $\mathrm{CO}$ emission, the main tracer for molecular gas (e.g., review by Carilli \& Walter 2013). Essentially all these detections were obtained by targeted observations of galaxies that had been pre-selected through their star forming properties (e.g., UV or FIR emission).

Advances in millimeter technology now allow spectral-line scans across full atmospheric windows. Scans over a broad frequency range now allow meaningful attempts at redshift determinations in heavily obscured, high- $z$ galaxies, through the detection of molecular gas tracers. In turn, they enable studies of the molecular gas emission over large cosmic volumes. Such studies have been out of reach until very recently, because of limitations both in sensitivity and instantaneous frequency coverage of existing millimeter facilities. Here we present the first blind molecular line scan of an optical deep field, i.e., a region in the sky for which superb multi-wavelength data is available. Our target of choice is a region in the Hubble Deep Field North (HDF-N; Williams et al. 1996), one of the best studied optical/NIR deep fields. We have used the IRAM Plateau de Bure Interferometer (PdBI) to scan the $3 \mathrm{~mm}$ atmospheric band, to search for $\mathrm{CO}$ transitions. This band is particularly well suited for CO surveys of high- $z$ galaxies, as it targets the lowest- $J$ CO transitions, which are collisionally excited by $\mathrm{H}_{2}$ at low temperatures. As shown in Figure 1 and Table 1, the $\mathrm{CO}$ and $\left[\mathrm{C}_{\mathrm{I}}\right]$ redshift ranges probed in a $3 \mathrm{~mm}$ scan are $z \lesssim 0.5$, and $1.0 \lesssim z \lesssim 1.9$, and $z \gtrsim 2$. At $z \gtrsim 3$ two or more $\mathrm{CO}$ transitions are covered by such a scan, thus allowing unambiguous redshift identifications. In Walter et al. (2012), we used the dataset presented here to search for $\mathrm{CO}$ emission associated with HDF 850.1, the brightest submillimeter galaxy (SMG) in the HDF-N (Williams et al. 1996; Hughes et al. 1998). This search allowed us to unambiguously establish the redshift of this luminous galaxy $(z=5.183$; Walter et al. 2012). A 


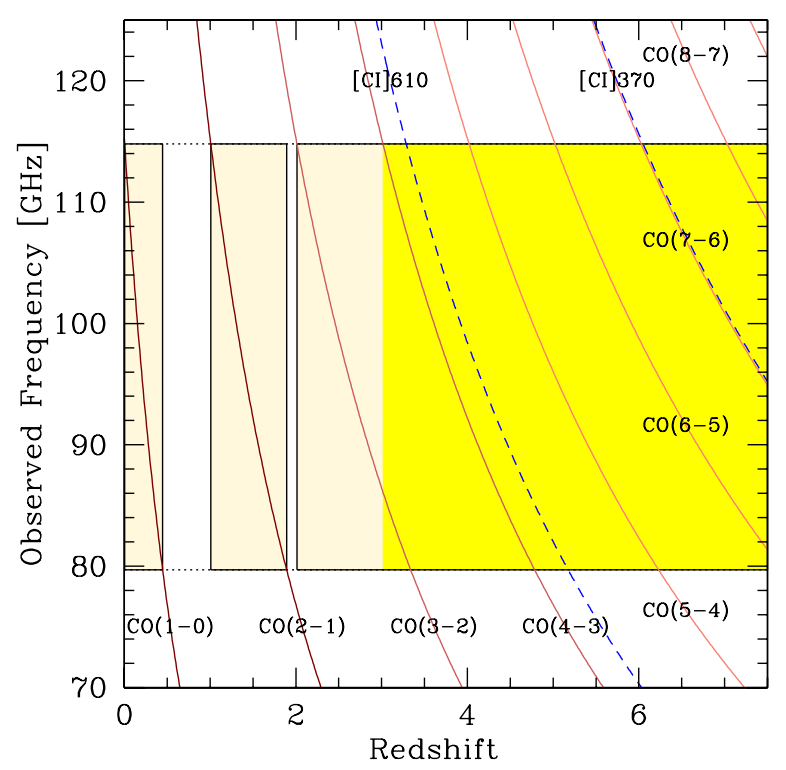

Figure 1. CO redshift coverage of our molecular line scan (from 79.7 to $114.8 \mathrm{GHz}$ ). We sample CO emission at $0.00412<z<0.4464,1.008<$ $z<1.893$, and at any $z>2.012$, as highlighted by the shaded area. Darker shading marks the redshift range $(z>3.016)$ for which multiple lines $(\mathrm{CO}$ or $[\mathrm{C} \mathrm{I}])$ are simultaneously covered.

(A color version of this figure is available in the online journal.)

Table 1

Lines and Corresponding Redshift Ranges Covered in Our Molecular Line Scan (79.7-114.8 GHz)

\begin{tabular}{lclccc}
\hline \hline Transition & $\begin{array}{c}v_{0} \\
(\mathrm{GHz})\end{array}$ & $z_{\min }$ & $z_{\max }$ & $\langle z\rangle$ & $\begin{array}{r}\text { Volume } \\
(2)\end{array}$ \\
$(1)$ & $(3)$ & $(4)$ & $(5)$ & $\begin{array}{c}(6) \\
(6)\end{array}$ \\
\hline $\mathrm{CO}(1-0)$ & 115.271 & 0.00412 & 0.446 & 0.338 & 92 \\
$\mathrm{CO}(2-1)$ & 230.538 & 1.008 & 1.893 & 1.525 & 1442 \\
$\mathrm{CO}(3-2)$ & 345.796 & 2.012 & 3.339 & 2.751 & 2437 \\
$\mathrm{CO}(4-3)$ & 461.041 & 3.016 & 4.785 & 3.981 & 2966 \\
$\mathrm{CO}(5-4)$ & 576.268 & 4.020 & 6.231 & 5.213 & 3249 \\
$\mathrm{CO}(6-5)$ & 691.473 & 5.023 & 7.676 & 6.445 & 3403 \\
$\mathrm{CO}(7-6)$ & 806.652 & 6.027 & 9.122 & 7.679 & 3484 \\
\hline$[\mathrm{C}]_{1-0}$ & 492.161 & 3.287 & 5.175 & 4.313 & 1550 \\
{$[\mathrm{C}]_{2-1}$} & 809.342 & 6.050 & 9.155 & 7.707 & 3508 \\
\hline
\end{tabular}

Notes. Comoving volume and volume-weighted average redshifts are computed by accounting for the changing size of the primary beam as a function of observed frequency.

similar "blind line search" approach was presented in Weiß et al. (2013), who performed a scan of the $3 \mathrm{~mm}$ window with the Atacama Large (Sub)Millimeter Array (ALMA) to obtain redshifts for 26 strongly lensed SMGs. This search resulted in the discovery of 44 lines, and in the identification of 20 unambiguous redshifts. The ALMA observations presented in Weiß et al. (2013) have a typical rms noise level of $2 \mathrm{mJy} \mathrm{beam}^{-1}$ per $50-60 \mathrm{~km} \mathrm{~s}^{-1}$ channel, i.e., a $4 \sigma$ line luminosity limit of $\sim 5 \times 10^{10} \mathrm{~K} \mathrm{~km} \mathrm{~s}^{-1} \mathrm{pc}^{2}$ (assuming a line width of $300 \mathrm{~km} \mathrm{~s}^{-1}$ ). This depth is insufficient to probe any $\mathrm{CO}$ emission arising from main-sequence galaxies in the targeted fields.

The molecular line scan ("blind CO search") presented here is the first one that is sensitive enough to probe the molecular content beyond the "tip of the iceberg" of luminous SMGs. This paper describes the scan and first observational results. In Section 2 we describe our observational setup. Section 3 presents the line-searching techniques adopted in our analysis. In

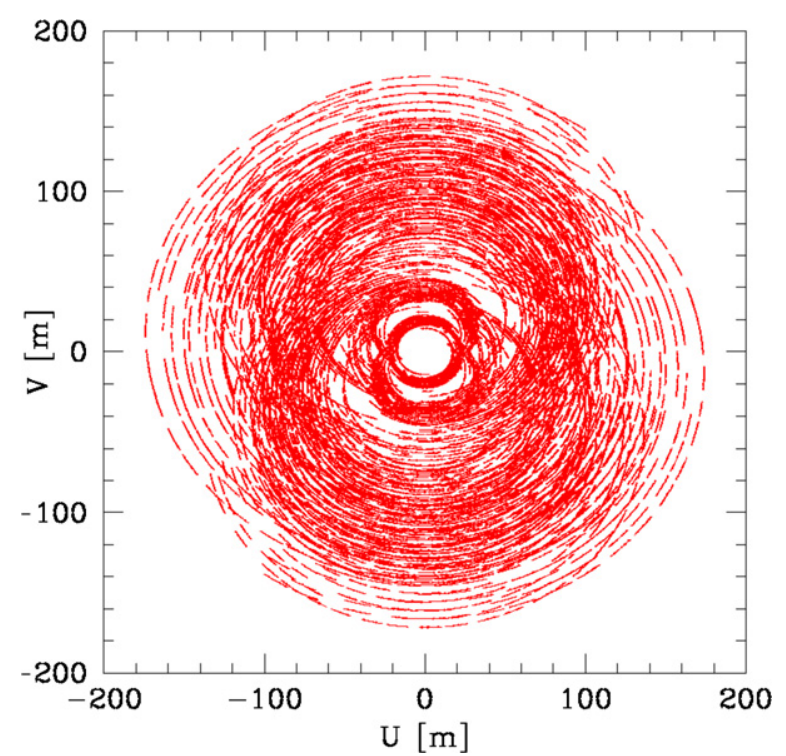

Figure 2. $(U, V)$ coverage of our observations. A total of 43 tracks and 132, 448 visibilities (110 hr on source, six-antenna equivalent) spread over 10 different frequency setups were used to produce the final data cubes.

(A color version of this figure is available in the online journal.)

Section 4 we describe our results from the line search, the properties of our $3 \mathrm{~mm}$ selected line candidates, and we investigate the CO properties of optically/NIR-selected galaxies. In Section 5 we compare our results with empirical predictions and Section 6 presents our analysis of the $3 \mathrm{~mm}$ continuum emission. Our conclusions are summarized in Section 7. Implications of our results regarding the cosmic abundance of molecular gas at high $z$ are presented in a companion paper (Walter et al. 2014; hereafter, W14).

Throughout the paper we will assume a standard cosmology with $H_{0}=70 \mathrm{~km} \mathrm{~s}^{-1} \mathrm{Mpc}^{-1}, \Omega_{\mathrm{m}}=0.3$ and $\Omega_{\Lambda}=0.7$.

\section{OBSERVATIONS}

\subsection{The $3 \mathrm{~mm}$ Scan}

We have obtained a continuous scan of the $3 \mathrm{~mm}$ window performed at the IRAM PdBI (pointing center: R.A. = 12:36:50.300, decl. $=+62: 12: 25.00, \mathrm{~J} 2000.0) .{ }^{15}$ The primary beam of PdBI can be described by a Gaussian profile with FWHM $=47^{\prime \prime} .3 \times(100 / v)$, where $v$ is the observing frequency in $\mathrm{GHz}$. At the central frequency of our scan $(v=97.25 \mathrm{GHz})$, the primary beam is 48 ". 6 in diameter. Observations were obtained between 2010 December 6 and 2012 November 19 split into 43 tracks (projects U09E, VOB6, V--3). We covered the entire $3 \mathrm{~mm}$ window (79.696-114.798 GHz) with 10 frequency settings (labeled with capital letters from $\mathrm{A}$ to $\mathrm{J}$ with increasing frequency) using the PdBI WideX correlator. Observations were obtained in $\mathrm{C}$ configuration, in most cases using the full six-antenna array, resulting in baselines from $14.5 \mathrm{~m}$ to $176 \mathrm{~m}$ (see Figure 2). Flux calibration was achieved by observing various calibrators (in most cases: MWC349, B0923+392, 3C84, 3C273, 3C 345, B0234+285, B2200+420, B1749+096). The quasar B1300+580 was used as a phase and amplitude calibrator. Data have been processed using the most recent version of the GILDAS software. The receiver operated in the lower sideband at frequencies

15 This particular region within the HDF-N was chosen to include the bright SMG HDF 850.1, see Walter et al. (2012). 


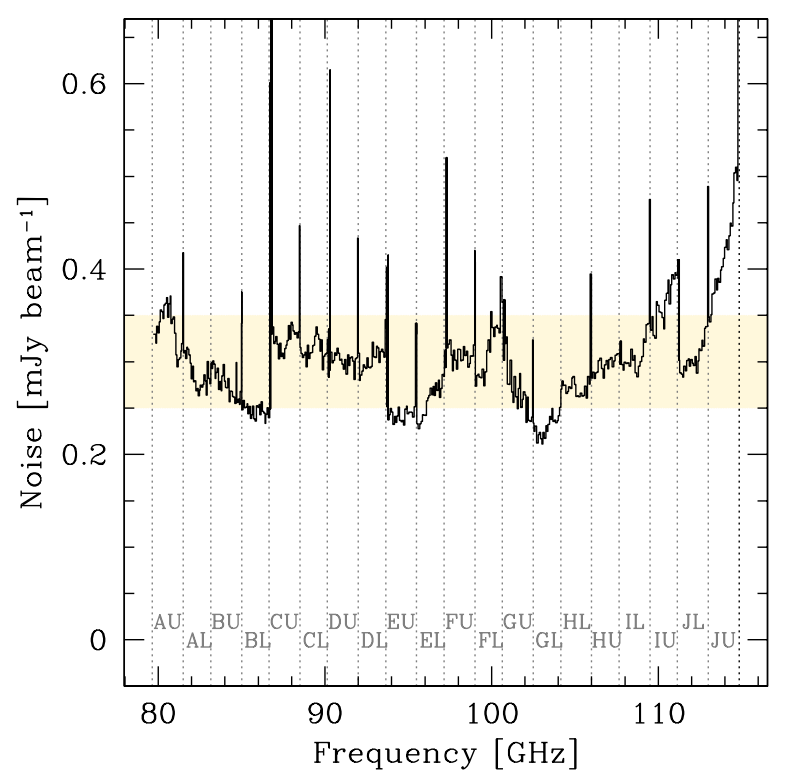

Figure 3. Noise per $90 \mathrm{~km} \mathrm{~s}^{-1}$ channel in our molecular line scan. The different frequency setups of our observations are labeled. Spikes in the noise properties are observed at the edges of various frequency setups. The shaded box highlights the $(0.25-0.35) \mathrm{mJy}_{\text {beam }}{ }^{-1}$ range, our typical sensitivity limit.

(A color version of this figure is available in the online journal.)

below 104.2 GHz (setups A-G) and in the upper sideband in the remaining part of the scan (setups $\mathrm{H}-\mathrm{J}$ ). WideX has four different units, two observing the two polarization modes on the low-frequency side of the tuning frequency, and two observing the high-frequency side. We therefore extracted two cubes (at " $U$ " and " $L$ " with respect to the tuned frequency) for each frequency setup. We resampled the cubes in $90 \mathrm{~km} \mathrm{~s}^{-1}$ channels, and imaged them using the GILDAS suite mapping. Natural weighting was adopted.

The typical beam size in our molecular line scan is $3^{\prime \prime} \times 2^{\prime \prime}$.7 $(\sim 25 \mathrm{kpc}$ at $z=2$, and roughly constant at any $z \gtrsim 1)$. The final cubes include 132, 448 visibilities in total, corresponding to $\approx 110 \mathrm{hr}$ on source (six-antenna equivalent). The final cubes have a typical rms of $0.3 \mathrm{mJy}^{\mathrm{beam}}{ }^{-1}$ in $90 \mathrm{~km} \mathrm{~s}^{-1}$ channels and we show the actual frequency dependence of the noise in Figure 3. We can convert this limit into a CO luminosity sensitivity, by assuming a typical line width of $300 \mathrm{~km} \mathrm{~s}^{-1}$, and an average primary beam correction of 0.72 , and by requiring a $3.5 \sigma$ line detection. The resulting $\mathrm{CO}$ luminosity limit as a function of redshift is shown in Figure 4. At $z>1$, our $3.5 \sigma$ CO line detection threshold is $L_{\lim }^{\prime}=(4-8) \times 10^{9} \mathrm{~K} \mathrm{~km} \mathrm{~s}^{-1} \mathrm{pc}^{2}$, comparable with the faintest $\mathrm{CO}$ detections reported so far from $3 \mathrm{~mm}$ observations at these redshifts (Carilli \& Walter 2013).

\subsection{Follow-up Observations}

In addition to the $3 \mathrm{~mm}$ scan, we obtained additional observations at the PdBI in the $2 \mathrm{~mm}$ band to cover a higher- $J$ CO line of the brightest line candidate in our $3 \mathrm{~mm}$ scan (ID.03, below). These observations were obtained between 2011 December 15 and 28 (four tracks), with the array in six-antenna compact configuration ("special"). Baselines ranged between $19.0 \mathrm{~m}$ and $98.1 \mathrm{~m}$. The pointing center was R.A. = 12:36:49.10, decl. = $+62: 12: 11.3$ (J2000.0), i.e., 5".6 away from the position of the $3 \mathrm{~mm}$ line. The observing frequency was tuned to $166.500 \mathrm{GHz}$, encompassing the $\mathrm{CO}(4-3)$ transition at $z=1.784$. The final cube includes 7469 visibilities, corresponding to $6.22 \mathrm{hr}$ on source. The beam is $3^{\prime \prime} .5 \times 2$.' 6 , and the noise per $90 \mathrm{~km} \mathrm{~s}^{-1}$

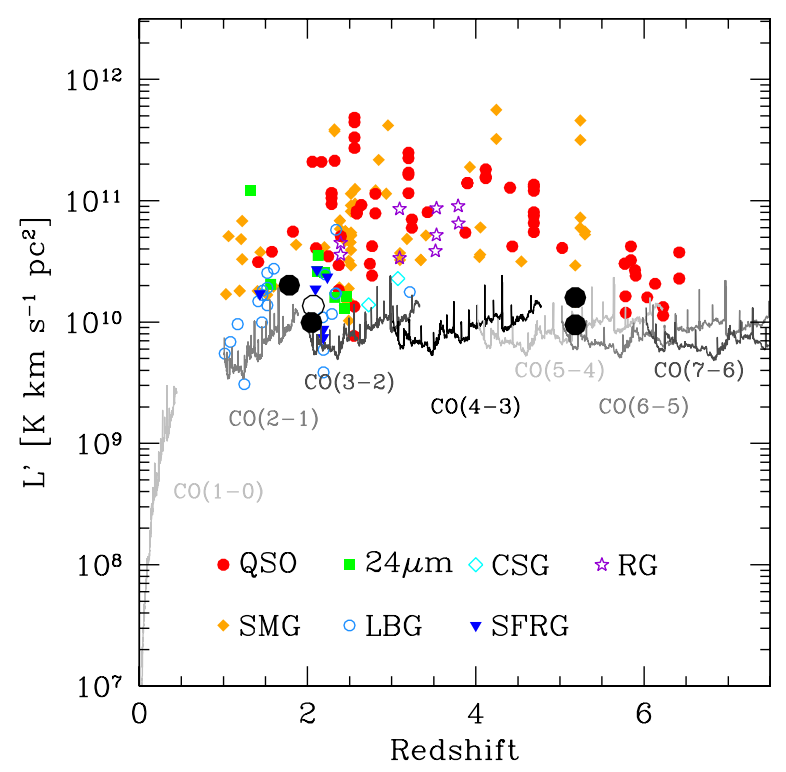

Figure 4. Sensitivity of our $3 \mathrm{~mm}$ scan in terms of line luminosity, for various $\mathrm{CO}$ transitions. The luminosity sensitivity is computed by assuming a line width of $300 \mathrm{~km} \mathrm{~s}^{-1}$, a $3.5 \sigma$ detection (see Section 2) and an average primary beam attenuation of 0.72 . The big filled circles mark the two $\mathrm{CO}$ detections from HDF 850.1 at $z=5.183$ obtained in our molecular line scan, the $\mathrm{CO}(2-1)$ detection in ID.03 (the brightest line in our sample), and the $\mathrm{CO}(3-2)$ detection of ID.19, the only $\mathrm{CO}$ line in our survey for which a consistent spectroscopic redshift from the optical/NIR counterpart is available. The big empty circle marks the CO detection of ID.18, the only other line detected at $>5 \sigma$, for which we assume here a redshift $z=2.070$. Our observations are sensitive to CO line luminosities (4-8) $\times 10^{9} \mathrm{~K} \mathrm{~km} \mathrm{~s}^{-1} \mathrm{pc}^{2}$ at any $z>1$. For the sake of comparison, we overplot all $z>1 \mathrm{CO}$ detections to date from $3 \mathrm{~mm}$ observations, from the compilation by Carilli \& Walter (2013) (here we have not applied de-magnification corrections). Colors and shapes of symbols mark different source types (quasars, submillimeter galaxies, $24 \mu \mathrm{m}$ selected galaxies, Lyman-break galaxies, color-selected galaxies, star-forming radio galaxies, radio galaxies). With our achieved sensitivity, we would be able to detect essentially all previously detected $\mathrm{CO}$ emitters at high redshift.

(A color version of this figure is available in the online journal.)

channel is $0.96 \mathrm{mJy}$ beam $^{-1}$. In addition, the $\mathrm{CO}(1-0)$ transition of this line candidate was targeted with the JVLA in $Q$ band $(41.39 \mathrm{GHz})$ in $\mathrm{C}$-array, resulting in a resolution of 0.6 (noise of $0.09 \mathrm{mJy}$ over a channel width of $45.5 \mathrm{MHz}$, i.e., the full $\mathrm{CO}(2-1)$ line width). These observations are discussed in Section 4.5 (ID.03) and the corresponding figure (Figure 11).

\section{LINE SEARCH APPROACH}

The main goal of this project is to blindly search for line emission in our scan, with no pre-selection based on optical/ NIR observations. In the following we discuss the line-searching algorithms used in this analysis.

\subsection{The spread Analysis}

Our first method is based on the noise statistics in each channel. Typically, pixel values in each channel map have a Gaussian distribution, the width of which is set by the noise level at that frequency. ${ }^{16}$ If a bright, point-like source is present at a given frequency (redshift), a few pixels will

\footnotetext{
16 Formally, the pixels are spatially correlated. For example, a single, highly deviating visibility would produce regular stripes in the image, thus resulting in an excess of bright positive and negative pixels. Moreover, our resolution element is $\sim 4$ times larger than the pixel size. Nevertheless, the analysis described here does not rely on a probabilistic interpretation of the pixel distribution, and is only marginally affected by the number of pixels per resolution element.
} 

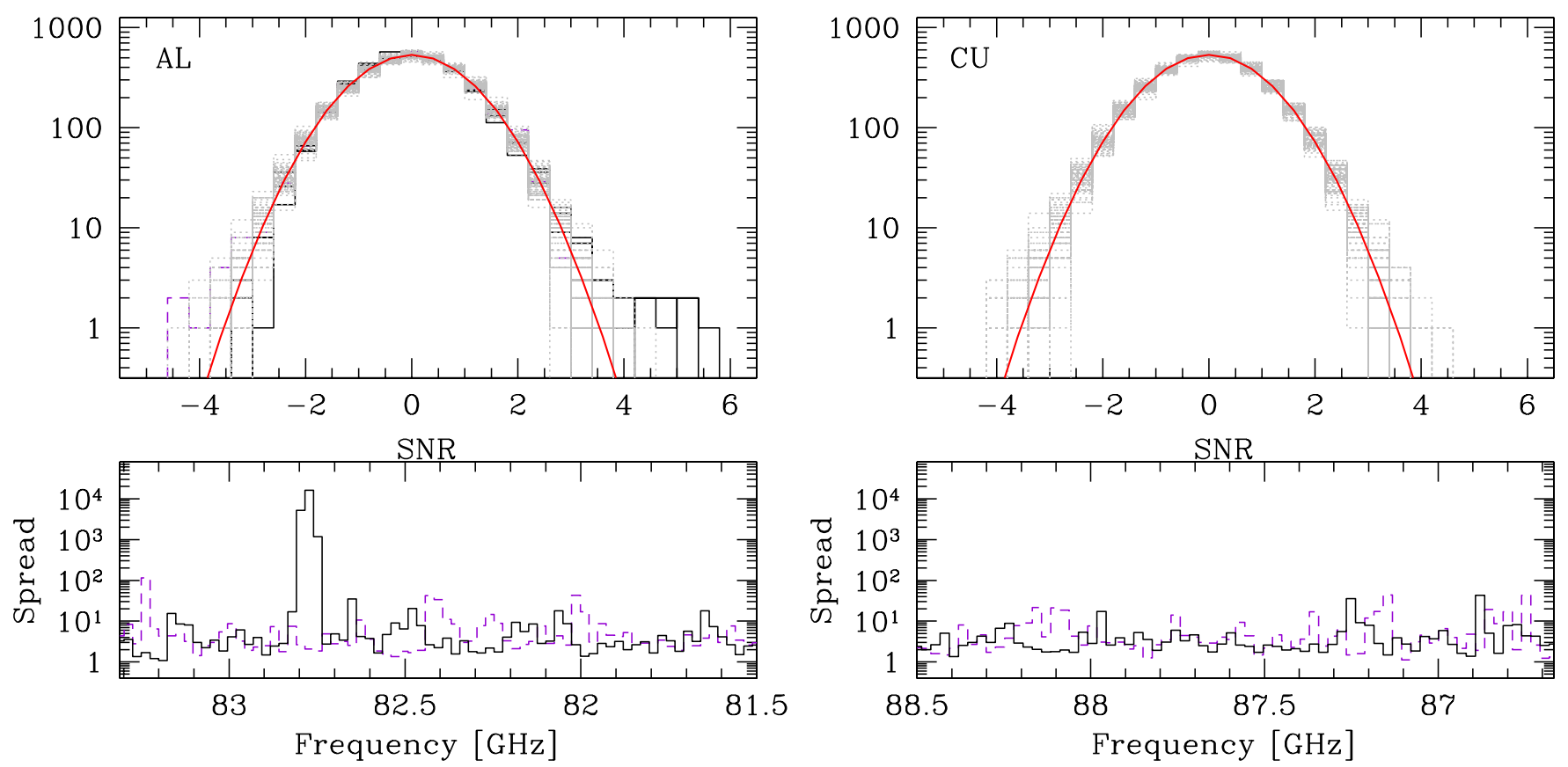

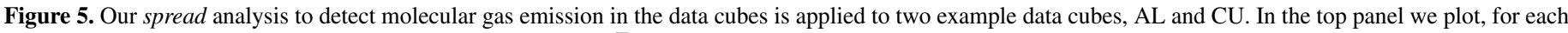

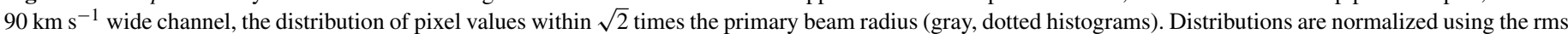

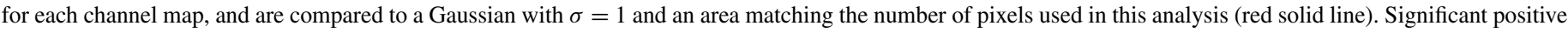

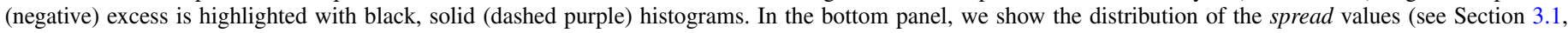

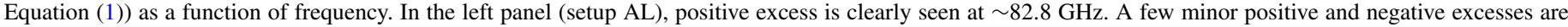
also reported in the AL cube. No significant deviations from a purely Gaussian distribution is reported in the CU cube, implying no line detection.

(A color version of this figure is available in the online journal.)

show a clear deviation from the Gaussian distribution, thus introducing a wing toward high signal-to-noise ratios $(\mathrm{S} / \mathrm{Ns})$ of the distribution. We quantify this deviation by defining a "spread" parameter:

$$
\text { spread }=\Sigma_{0}^{ \pm \infty} f_{\text {obs }}(\mathrm{S} / \mathrm{N}) / f_{\exp }(\mathrm{S} / \mathrm{N}) .
$$

Here $f_{\text {obs }}$ is the observed distribution of pixel values, in units of $\mathrm{S} / \mathrm{N}$, while $f_{\exp }$ is the theoretical Gaussian (i.e., a Gaussian with $\sigma=1$ and an integrated area equal to the number of pixels). In this step we consider only pixels within $\sqrt{2} \times$ the primary beam's half width at half maximum (i.e., twice the area of the primary beam). The sum is computed from zero to $+\infty$ (i.e., tracing positive excesses) and from zero to $-\infty$ (i.e., tracing negative excesses). The latter is used to evaluate the purity of our selection (while positive excesses may be attributed either to real sources or noise, negative excesses are only due to noise). Due to its formulation, the spread index defined in Equation (1) is formally reminiscent of Pearson's $\chi^{2}$. We regularize channelby-channel variations by applying a box-car smoothing over three channels $\left(270 \mathrm{~km} \mathrm{~s}^{-1}\right)$ before computing the spread index. Figure 5 shows an example of a cube with a clear line detection (left panel), and of an "empty" cube with no significant line candidate (right panel).

Our spread analysis is a simple but effective hypothesis test. Compared with other statistical tools (e.g., $\chi^{2}$ test, Kolmogorov-Smirnov, Cramér-von Mises, etc.), the spread index has the advantage of down-weighting the central part of the distribution (i.e., many pixels with a measured flux around zero) and highlighting the presence of outliers in the pixel value distribution. Its applicability is limited to data sets for which we have an accurate analytical description of the noise properties $\left(f_{\text {exp }}\right)$. Such a requirement is generally satisfied in our data set (only a couple of channels over the whole scan are affected by receiver "parasites," which introduce strong non-Gaussian components in the noise pattern). However, this method is not sensitive to the position of the excess pixels in the map as no spatial coherence is required. For instance, a peak in the spread distribution as a function of the observed frequency may be associated with a single source (as in the case shown in Figure 5, left), or two sources, or more. We have visually inspected all the peaks revealed by the spread analysis, and progressively lowered the threshold above which a peak in the spread distribution was considered (spread $=15$ ). The same threshold has been adopted for negative candidates. ${ }^{17}$

\subsection{The cprops Search}

In parallel, we have run a different line-searching algorithm based on the finder cprops which has originally been designed to identify giant molecular clouds in nearby galaxies (Rosolowski \& Leroy 2006). This algorithm first computes the noise in the maps through the data cube, and divides the observed fluxes by the same value, so that data are stored in units of $\mathrm{S} / \mathrm{N}$. A mask is associated with any pixel exceeding a given $\mathrm{S} / \mathrm{N}$ threshold in two consecutive channels. This mask looks for the "core" in the profiles of line candidates. A second mask is generated by selecting the pixels that have a positive excess (down to a lower threshold) in two neighboring channels. This second mask thus identifies the wings of the lines. If the masks overlap in more than two consecutive channels, the excess is considered

\footnotetext{
17 We note that, since we are dealing with dirty cubes, bright sources would introduce both positive and negative pixels. However, even in cases of modest $(\mathrm{U}, \mathrm{V})$ coverage, negative pixels due to secondary lobes in the beam shape are typically much less prominent (by a factor $\gtrsim 3$ ) than the central positive peak associated with a point source. Therefore, their imprint on the spread distribution will be minimal and will not affect our search.
} 
Table 2

Catalog of the Line Candidates Identified in Our Analysis, Ordered by Frequency

\begin{tabular}{|c|c|c|c|c|c|c|c|c|c|}
\hline (1) & $\begin{array}{c}\text { R.A. } \\
(\mathrm{J} 2000.0) \\
(2)\end{array}$ & $\begin{array}{c}\text { Decl. } \\
(\mathrm{J} 2000.0) \\
(3)\end{array}$ & $\begin{array}{c}\text { Frequency } \\
(\mathrm{GHz}) \\
(4)\end{array}$ & $\begin{array}{c}\text { Flux } \\
\left(\mathrm{Jy} \mathrm{km} \mathrm{s}^{-1}\right) \\
(5)\end{array}$ & $\begin{array}{c}\mathrm{S} / \mathrm{N}_{\text {map }} \\
\text { (6) }\end{array}$ & $\begin{array}{c}S / N_{\text {spec }} \\
(7)\end{array}$ & $\begin{array}{c}\text { FWHM } \\
\left(\mathrm{km} \mathrm{s}^{-1}\right) \\
(8)\end{array}$ & $\begin{array}{c}\text { Quality } \\
\text { (9) }\end{array}$ & $\begin{array}{l}\text { Notes } \\
(10)\end{array}$ \\
\hline ID.01 & $12: 36: 47.72$ & $+62: 12: 19.0$ & $80.05 \pm 0.03$ & $0.531 \pm 0.116$ & 5.0 & 4.6 & $440_{-200}^{+250}$ & 1 & High-quality \\
\hline ID. 02 & $12: 36: 52.27$ & $+62: 12: 51.7$ & $82.07 \pm 0.02$ & $0.374 \pm 0.106$ & 4.3 & 3.5 & $130_{-40}^{+140}$ & 1 & High-quality \\
\hline ID.03 & $12: 36: 48.58$ & $+62: 12: 15.5$ & $82.80 \pm 0.02$ & $0.502 \pm 0.080$ & 7.3 & 6.3 & $330_{-40}^{+120}$ & 1 & Secure \\
\hline ID.04 & $12: 36: 52.56$ & $+62: 12: 35.0$ & $84.94 \pm 0.03$ & $0.189 \pm 0.070$ & 5.1 & 2.7 & $210_{-50}^{+230}$ & 2 & \\
\hline ID.05 & $12: 36: 52.37$ & $+62: 12: 11.1$ & $89.89 \pm 0.04$ & $0.473 \pm 0.112$ & 4.6 & 4.2 & $360_{-40}^{+80}$ & 1 & High-quality \\
\hline ID.06 & $12: 36: 49.91$ & $+62: 12: 27.2$ & $90.01 \pm 0.03$ & $-0.227 \pm 0.067$ & 4.6 & 3.4 & $240_{-140}^{+150}$ & 2 & Negative \\
\hline ID.07 & $12: 36: 47.04$ & $+62: 12: 41.7$ & $91.84 \pm 0.04$ & $-0.378 \pm 0.146$ & 3.7 & 2.6 & $290_{-40}^{+40}$ & 2 & Negative \\
\hline ID. 08 & $12: 36: 52.15$ & $+62: 12: 25.8$ & $93.17 \pm 0.03$ & $0.423 \pm 0.097$ & 4.4 & 4.4 & $490_{-50}^{+200}$ & 1 & HDF 850.1 \\
\hline ID.09 & $12: 36: 51.15$ & $+62: 12: 40.6$ & $93.91 \pm 0.04$ & $-0.194 \pm 0.073$ & 4.5 & 2.7 & $120_{-50}^{+150}$ & 2 & Negative \\
\hline ID. 10 & $12: 36: 49.22$ & $+62: 12: 25.7$ & $103.88 \pm 0.03$ & $0.188 \pm 0.044$ & 4.0 & 4.2 & $150_{-50}^{+50}$ & 1 & High-quality \\
\hline ID. 11 & $12: 36: 47.10$ & $+62: 12: 18.0$ & $108.32 \pm 0.03$ & $0.362 \pm 0.101$ & 4.0 & 3.6 & $150_{-40}^{+70}$ & 1 & High-quality \\
\hline ID. 12 & $12: 36: 49.57$ & $+62: 12: 19.9$ & $108.43 \pm 0.04$ & $0.262 \pm 0.074$ & 4.2 & 3.5 & $210_{-50}^{+150}$ & 1 & High-quality \\
\hline ID. 13 & $12: 36: 47.19$ & $+62: 12: 18.6$ & $108.77 \pm 0.03$ & $0.242 \pm 0.115$ & 4.1 & 2.1 & $140_{-50}^{+50}$ & 2 & \\
\hline ID. 14 & $12: 36: 50.85$ & $+62: 12: 30.1$ & $109.65 \pm 0.03$ & $0.244 \pm 0.072$ & 5.3 & 3.4 & $190_{-40}^{+50}$ & 2 & \\
\hline ID. 15 & $12: 36: 48.56$ & $+62: 12: 40.4$ & $109.79 \pm 0.03$ & $0.459 \pm 0.113$ & 4.6 & 4.1 & $260_{-50}^{+120}$ & 1 & High-quality \\
\hline ID. 16 & $12: 36: 50.49$ & $+62: 12: 16.1$ & $110.55 \pm 0.04$ & $-0.311 \pm 0.087$ & 3.6 & 3.6 & $240_{-40}^{+150}$ & 1 & Negative \\
\hline ID. 17 & $12: 36: 51.99$ & $+62: 12: 25.6$ & $111.85 \pm 0.06$ & $0.366 \pm 0.101$ & 5.4 & 3.6 & $370_{-120}^{+150}$ & 1 & HDF 850.1 \\
\hline ID. 18 & $12: 36: 48.79$ & $+62: 12: 38.0$ & $112.63 \pm 0.04$ & $0.584 \pm 0.115$ & 5.6 & 5.1 & $290_{-40}^{+80}$ & 1 & High-quality \\
\hline ID. 19 & $12: 36: 51.37$ & $+62: 12: 16.9$ & $113.45 \pm 0.04$ & $0.436 \pm 0.116$ & 4.3 & 3.8 & $350_{-40}^{+150}$ & 1 & Secure \\
\hline ID. 20 & $12: 36: 51.54$ & $+62: 12: 12.0$ & $113.45 \pm 0.05$ & $0.388 \pm 0.109$ & 3.6 & 3.6 & $150_{-70}^{+100}$ & 1 & High-quality \\
\hline ID. 21 & $12: 36: 50.48$ & $+62: 12: 05.8$ & $114.15 \pm 0.05$ & $0.360 \pm 0.132$ & 3.2 & 2.7 & $220_{-90}^{+180}$ & 2 & \\
\hline
\end{tabular}

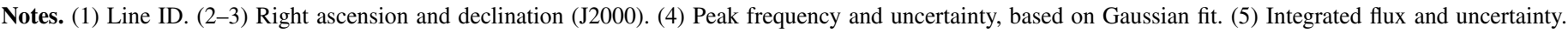

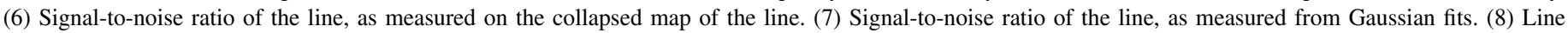

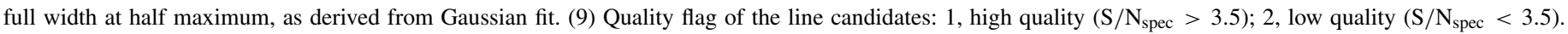
(10) Notes.

significant. Compared with the spread analysis, this approach has the advantage of using the spatial information available, but its purity is usually lower, unless very conservative significance cuts are adopted. Another difference is that the cprops search implicitly assumes Gaussian properties of the noise, while the spread analysis is in principle applicable to any distribution of data for which an analytical model of the noise is available.

\subsection{Selection of Line Candidates}

We ran the cprops algorithm by requiring a $>3 \sigma$ excess for the line core, and a $>1.5 \sigma$ excess in the neighboring channels. This search produced 117 positive line candidates and 101 negative ones. We visually inspected all of them, and crossmatched them with the results from the spread analysis. A large number of the sources were excluded because (1) the peak position observed in one channel drifts by a few arcseconds in the neighboring channels; or (2) the spread analysis does not reveal any significant excess. The final list of candidates includes only sources which have been selected by both methods. Our compilation thus consists of 17 positive and 4 negative line candidates (see Table 2 and Figure 6). Since our analysis deals with positive and negative line candidates in the same way, we expect about 4 false positive candidates out of 17 . The location of the line candidates in the HDF-N is shown in Figure 7.

L. Lentati et al. (in preparation) have developed a completely independent line-searching technique which relies on Bayesian inference. This method computes the probability that a given feature in the data is a real galaxy, based on a source model (e.g., expected line profiles, physical sizes, fluxes). This search confirmed the detection of all the brightest $\left(>0.4 \mathrm{Jy} \mathrm{km} \mathrm{s}^{-1}\right)$ lines identified in our search. The most significant line candidate found by Lenatati et al. that is not included in the present paper is at R.A. $=12: 36: 48.32$, decl. $=+62: 12: 38.44, v=89.28 \mathrm{GHz}$. This line candidate is observed in the spread analysis, but is not selected by cprops since in no individual channel the line candidate exceeds the $3 \sigma$ threshold. For consistency we exclude this candidate in the remainder of our analysis.

\subsection{Completeness and Purity from Artificial Data Cubes}

In order to estimate the completeness, degree of purity, and role of the Eddington bias in our line searches, we generated data cubes from artificial visibilities with the same format (i.e., UV-coverage, spectral setup, number of visibilities, etc.) of a typical data cube in our observations. We included Gaussian noise on the real and the imaginary parts of each visibility, resulting in a rms of $0.3 \mathrm{mJy} \mathrm{beam}^{-1}$ per $90 \mathrm{~km} \mathrm{~s}^{-1}$ channel. We then added 10 sources that are randomly distributed over a region twice as big as the primary beam. Each source represents a point source with an emission line peaked at a random frequency within the cube, and no continuum emission. The line profile is assumed to be Gaussian, with a FWHM $=300 \mathrm{~km} \mathrm{~s}^{-1}$, and peak flux set to $3.0 \mathrm{mJy}$ (scaled down according to the primary beam attenuation). We generated 10 realizations of each cube (i.e., 100 sources in total). We then ran our line-searching algorithms, and counted the number of input sources that have been successfully recovered. The whole process was repeated for sources with 

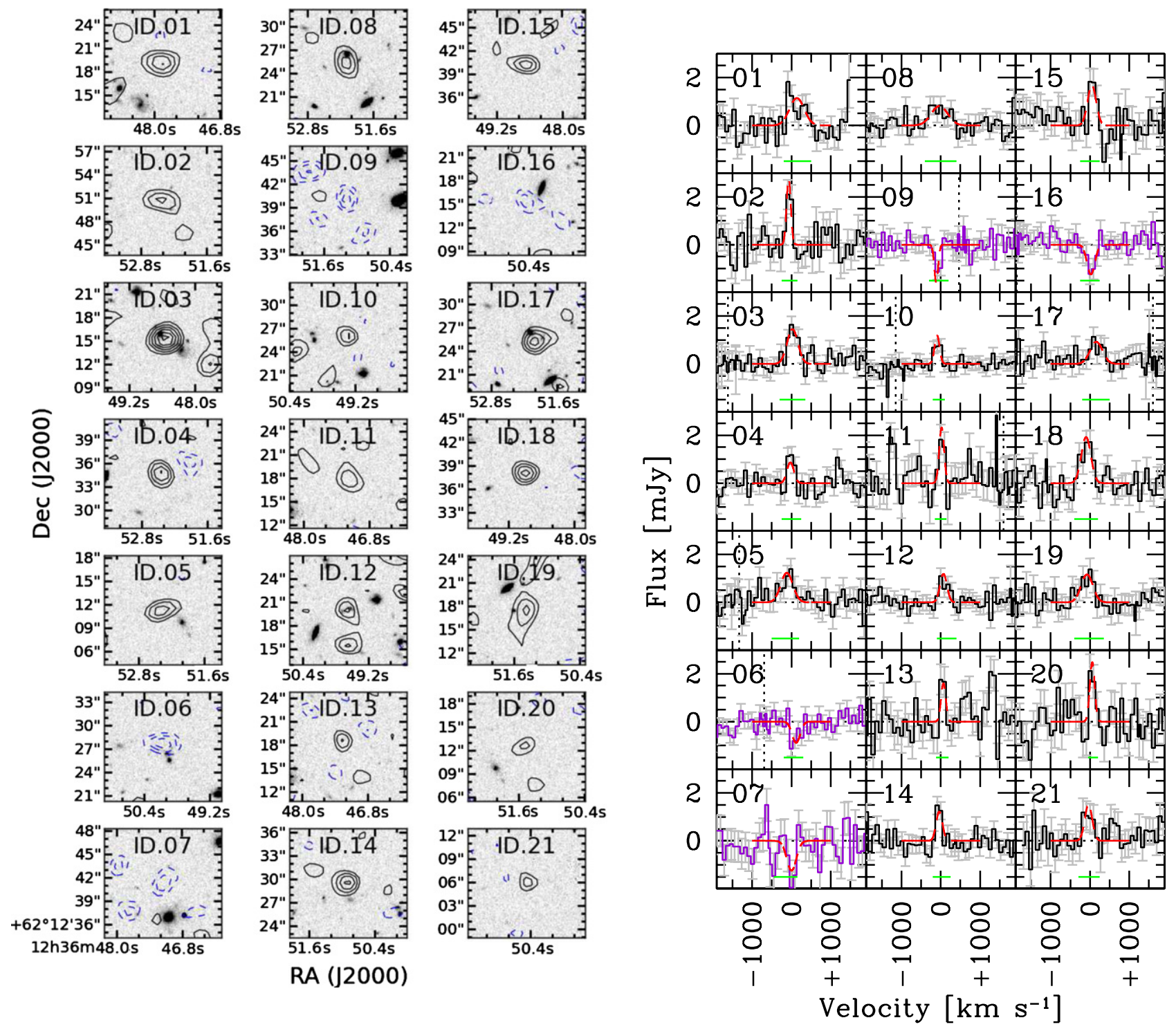

Figure 6. Line maps (left) and spectra (right) of the line candidates revealed by our search (Table 2). Left: line maps are shown as contours (solid black/dashed blue contours show positive/negative $[2,3,4, \ldots] \times \sigma$ emission). The gray scale shows $H S T$ /WFC3 F160W cutouts of the field from the CANDELS survey (Grogin et al. 2011; Koekemoer et al. 2011), centered at the coordinates of the line candidate (each region is $14^{\prime \prime} \times 14^{\prime \prime}$ wide). Right: spectra at the position of the line detections are corrected for primary beam attenuation. Solid histograms with error bar show the observed spectra. Negative line candidates are plotted in violet. The dotted black line shows the zero level, the dashed red line shows the best Gaussian fit (numbers are recorded in Table 2). The horizontal green bar shows the channels used in the line fits.

(A color version of this figure is available in the online journal.)

peak flux $=2.5,2.0,1.5,1.0$, and $0.5 \mathrm{mJy}$. Figure 8 shows the results of this analysis. We find that $>50 \%$ of the input sources within the primary beam are recovered for line peak fluxes of $1.5 \mathrm{mJy}$. The completeness increases at decreasing distance from the pointing center, and at increasing fluxes: $\sim 66 \%$ at $1.5-2.0 \mathrm{mJy}$ (comparable with the typical fluxes of the sources in Table 2) and reaches up to $85 \%$ for line peak fluxes $\gtrsim 2.5 \mathrm{mJy}$.

In each realization, we then counted the number of sources identified by the spread analysis, by cprops, and by both, which do not match any input source. These detections allow us to estimate the rate of false positives in our sample. We find that, on average, the spread analysis identifies 5.2 false positives per cube, cprops about 2.5 , and the combination of the two only 0.1 false positive per cube. This implies that, out of the whole scan (equivalent to 20 cubes), we expect $\sim 2$ false positives. This estimate is in agreement with the number of negative peaks identified as potential sources in Section 3.3.

\section{RESULTS}

\subsection{Properties of $3 \mathrm{~mm}$ Selected Line Candidates}

Figure 6 shows the line spectra and maps of the 21 line candidates discovered in our study (including "negative" candidates, which are used to gauge the purity of our line search). Line maps are extracted by integrating over the channels encompassing the full width at zero flux of each line candidate. We compute the map-based $\mathrm{S} / \mathrm{N}$ of each line as the ratio between the line flux (i.e., the value of the brightest pixel in the line map, in Jy beam ${ }^{-1}$ ) and the rms as measured in the line map (see Table 2). Then, for each line, we extract the spectra at the 


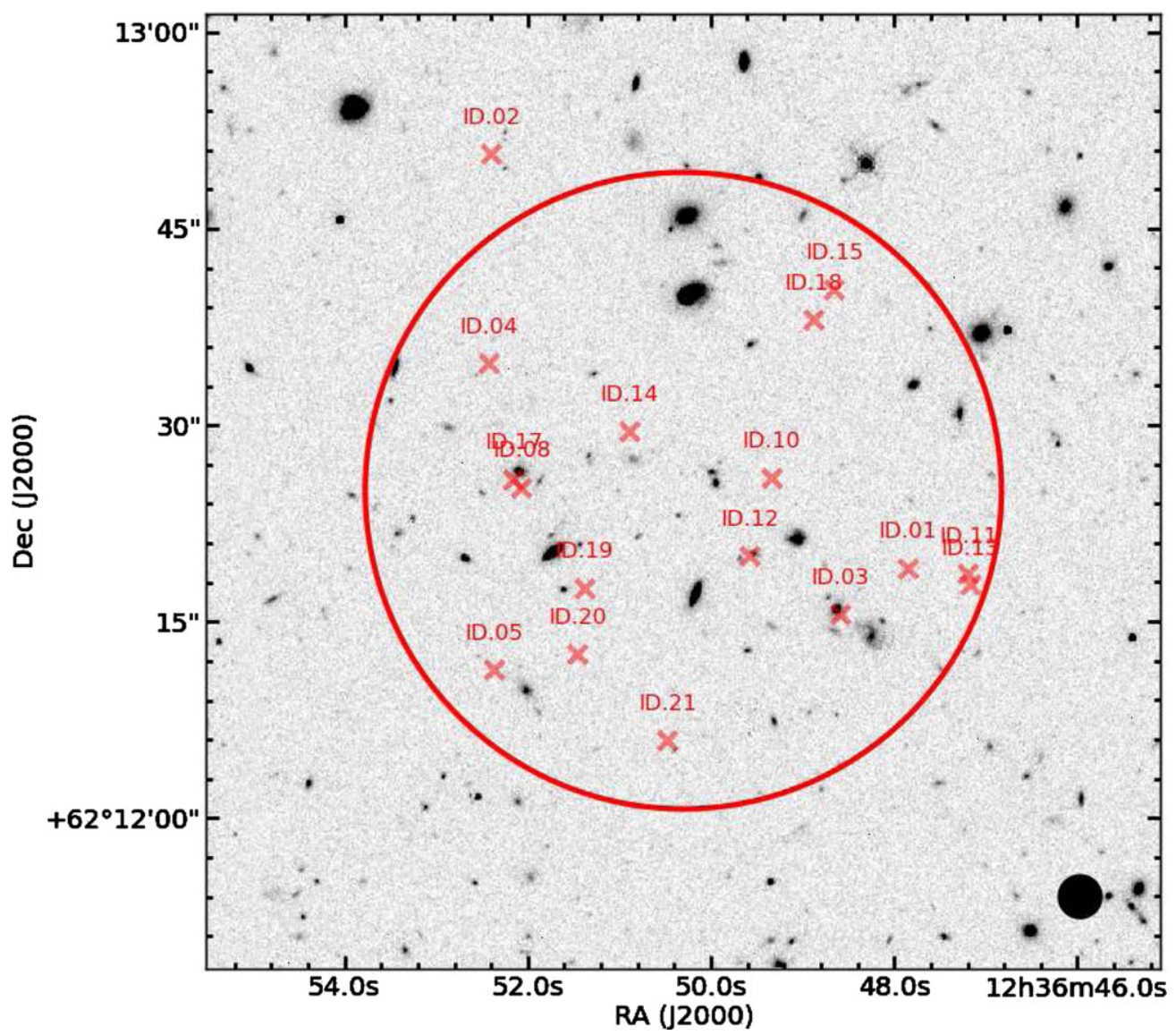

Figure 7. HST/WFC3 F160W (1.6 $\mu \mathrm{m}$, gray-scale) image of the region of the HDF-N studied here, from the CANDELS survey (Grogin et al. 2011; Koekemoer et al. 2011). The big circle shows the primary beam of our observations at the central frequency of our scan $(97.25 \mathrm{GHz})$. The positive line candidates discovered in our searches are plotted with red crosses (from Table 2). The typical beam size of our observations is shown as a filled black circle in the bottom right.

(A color version of this figure is available in the online journal.)

coordinates corresponding to the brightest pixel of each line candidate. ${ }^{18}$ Spectra are then corrected for primary beam attenuation, assuming a Gaussian attenuation and the frequency dependence of the primary beam as described in Section 2. The $\mathrm{S} / \mathrm{N}$ of the line candidates is usually modest, therefore a proper characterization of the line profiles is not possible. In most of the cases, however, a Gaussian fit provides a good description of the observed lines. We assume no underlying continuum emission, justified by the analysis of our continuum image (see Section 6). The measured line fluxes range between 0.16 and $0.56 \mathrm{Jy} \mathrm{km} \mathrm{s}^{-1}$. Line widths, as derived from the Gaussian fits, range between 110 and $500 \mathrm{~km} \mathrm{~s}^{-1}$ with significant uncertainties. The spectrum-based $\mathrm{S} / \mathrm{N}$ is computed as the ratio between the fitted line flux and its uncertainty. ${ }^{19}$

Based on the spectral S/N of the line candidates, we define a quality flag: We consider high-quality candidates (quality flag: 1) objects with $\mathrm{S} / \mathrm{N}_{\text {spec }}>3.5$ (14 candidates), and we label as low-quality candidates (quality flag: 2 ) those with $\mathrm{S} / \mathrm{N}<3.5$ (7 candidates). We note that three out of four of the negative peaks belong to the "low-quality" class, while at

\footnotetext{
18 We note that this approach may slightly underestimate the significance of real spectral features, because of the (relatively big) uncertainties in the coordinates of line baricenters.

$19 \mathrm{~S} / \mathrm{N}_{\text {spec }}$ is lower than the map-based $\mathrm{S} / \mathrm{N}$, because of model uncertainties (lines not being well described by a single Gaussian) and because the Gaussian fits also include the line wings, which worsen the noise, without contributing significant flux, while the line map is extracted only in channels where the line is clearly detected.
}

least two lines in the "high-quality" class are known to be real (the two CO transitions associated with HDF 850.1).

\subsection{Assigning Redshifts to Line Candidates}

The most prominent lines that we expect to detect in the $3 \mathrm{~mm}$ band are various $\mathrm{CO}$ transitions (up to $J_{\text {up }} \sim 7$ ) and the neutral carbon fine-structure lines $\left[\mathrm{CI}_{1-0}\right.$ and $\left[\mathrm{CI}_{1}\right]_{2-1}$ (see Table 1 and Figure 1). In the following subsections, we assign the most likely redshift (and thus CO transition) for each line candidate. In Figure 9 we provide a sketch of our approach to identify which $\mathrm{CO}$ transition (and thus redshift) the respective line candidates correspond to. First, we look for multiple transitions arising at the same spatial position (which would immediately constrain the redshift). We then search for an optical/NIR counterpart in the deep ancillary database of the HDF-N. If none is found, we identify the line candidate as the highest- $J$ CO transition consistent with the lack of multiple lines in the $3 \mathrm{~mm}$ window (i.e., we locate the line at the highest $z_{\mathrm{CO}}<3.016$ ). If one or more optical/NIR counterparts are available, we check whether a spectroscopic redshift is available and consistent with the line frequency observed in the $3 \mathrm{~mm}$ scan. If no spectroscopic information is available, we select plausible $\mathrm{CO}$ identifications on the basis of the UV-to-FIR spectral energy distributions (SEDs) of the counterparts. In the case of remaining ambiguity, we choose to adopt the CO redshift for which the SED fitting predicts the brightest FIR emission. This is justified by the observed correlation between FIR and CO luminosity (see, e.g., 


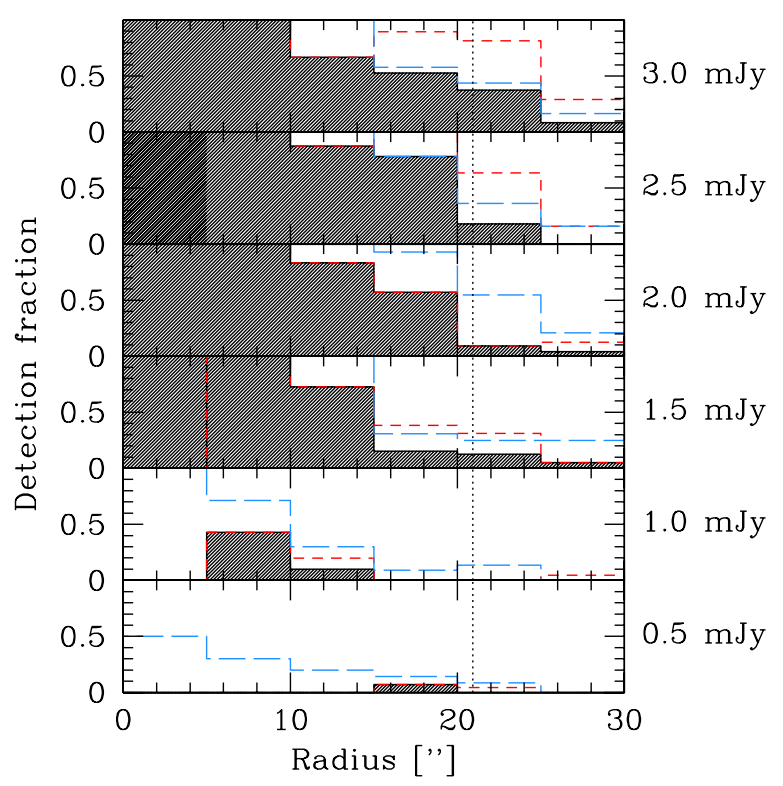

Figure 8. Completeness (detection fraction) of our line-searching techniques as a function of input source flux and offset with respect to the pointing center. The shaded histograms show the fraction of input sources that we successfully recovered with the combined use of the spread analysis and with cprops. The completeness levels achieved by applying only one of the two approaches are shown as empty histogram (blue, long-dashed = spread; red, short-dashed = cprops). The vertical dotted line shows the primary beam radius at the central frequency of the test cube. At the level of flux densities of interest here, we reach $>66 \%$ completeness.

(A color version of this figure is available in the online journal.)

Carilli \& Walter 2013): The SED model predicting the brightest dust emission also predicts brighter $\mathrm{CO}$ emission, which would increase the chances to detect a line in our scan. In the following sections, we discuss in more details the individual steps of this approach.

\subsection{Search for Multiple CO Transitions}

If a source is at $z>3.016$, at least two $\mathrm{CO}$ or [CI] lines are covered, providing an accurate redshift. ${ }^{20}$ In Figure 10 we show our basic diagnostic diagram for line identification, applied to two line candidates. In one case (line candidate ID.01), there is only one line observed in the $3 \mathrm{~mm}$ scan, thus excluding any $z>3$ identification (unless an extremely steep CO excitation ladder is invoked). The second case shown in Figure 10 has two lines (both independently identified in the line search process described in Section 3: lines ID.08 and ID.17). These are associated with the SMG HDF 850.1 and they are consistent with being $\mathrm{CO}(5-4)$ and $\mathrm{CO}(6-5)$ at $z \approx 5.18$ (Walter et al. 2012). HDF 850.1 is the only source for which we detected more than one line in the molecular line scan. Another pair of line candidates (ID.11 and ID.13) are also spatially consistent, but their frequency difference cannot be explained by any combination of $\mathrm{CO}$ or $[\mathrm{C} \mathrm{I}]$ lines at a single redshift. Therefore, we will treat these two lines as independent sources. All the remaining candidates show only one transition in the scan, thus suggesting that they are at $z \lesssim 3$.

\footnotetext{
20 We note that some ambiguity may persist at these low $\mathrm{S} / \mathrm{Ns}$, as $\mathrm{CO}$ transitions have frequencies which are $v_{0}\left[\mathrm{CO}\left(J_{\text {up }}-J_{\text {up }-1}\right)\right] \approx J_{\text {up }} \times$ $v_{0}[\mathrm{CO}(1-0)]$. For instance, two lines identified as $\mathrm{CO}(6-5)$ and $\mathrm{CO}(4-3)$ at $z_{1}$ can also be identified as $\mathrm{CO}(3-2)$ and $\operatorname{CO}(2-1)$ at $z_{2}=\left(z_{1}-1\right) / 2$. However, in the $z_{2}$ scenario the $\mathrm{CO}(5-4)$ line should also be detected, thus allowing us to discriminate between the two cases.
}

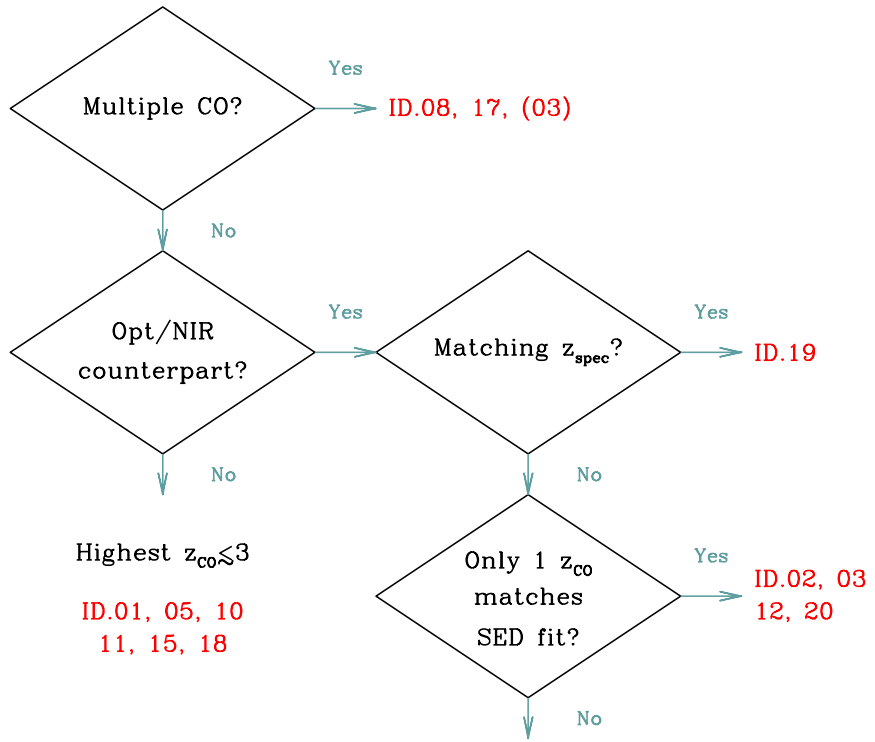

Bright FIR?

Figure 9. Scheme adopted in order to assign a $\mathrm{CO}$ transition (and thus redshift) to each of the line candidates of the molecular scan. We first look for multiple CO transitions in the $3 \mathrm{~mm}$ scan (implying a redshift $z \gtrsim 3$, see Figure 1 ); then we search for counterparts at optical/NIR wavelengths. If none is found (implying a faint/obscured/high- $z$ candidate galaxy), we assign the highest $z$ consistent with only one CO transition in the $3 \mathrm{~mm}$ window to the galaxy. If a counterpart is available, we check whether it has a spectroscopic redshift consistent with $z_{\mathrm{CO}}$; if not, we base our CO identification on the goodness of the SED fits. If none of the above applies, we opt for the $z_{\mathrm{CO}}$ for which the SED fit predicts the brightest FIR emission. The high-quality line candidates in our study are labeled in red. ID.03 is reported in bracket as a "multiple CO" identification, since the redshift is confirmed by other $\mathrm{CO}$ observations $(\mathrm{CO}(1-0)$ at $41.4 \mathrm{GHz}$ and $\mathrm{CO}(4-3)$ at $165.5 \mathrm{GHz}$ ), but they are not part of the original $3 \mathrm{~mm}$ scan (Section 2.2).

(A color version of this figure is available in the online journal.)

\subsection{Optical/NIR Counterparts of CO Line Candidates}

As a next step we search for counterparts at optical/NIR wavelengths of the CO line candidates. We take the HDF-N catalog of galaxies as reference (Williams et al. 1996), and we refer to the compilation of photometric redshifts by FernándezSoto et al. (1999). We also compare the positions of the line candidates in our survey with the IRAC and MIPS maps of this region (PI: M. Dickinson). Given the modest $\mathrm{S} / \mathrm{N}$ of our line detections, the uncertainties on the spatial position of the sources are comparable to the size of the resolution element (i.e., a radius of $\sim 1^{\prime \prime} .5$ ). Here we consider as tentative counterparts those galaxies that are within 1.5 of the peak position of the CO line candidates. Ten line candidates have one or more associations in the catalog by Fernández-Soto et al. (1999). One of them is a negative feature in our $3 \mathrm{~mm}$ scan (ID.06) and will be ignored in this analysis. Both ID.08 and ID.17 (the two transitions identified with HDF 850.1) are spatially consistent with a $z \approx 1$ galaxy; however, the redshift inferred by our $3 \mathrm{~mm}$ scan rules out such an association, as confirmed by the [C II] observations at higher spatial resolution presented in Walter et al. (2012).

The remaining seven $\mathrm{CO}$ line candidates have reliable galaxy associations. We collect photometric information of these sources from the catalogs by Williams et al. (1996) (ubvi), Dickinson et al. (2003) ( $H$ band), Bundy et al. (2009) (bvizK), M. Dickinson et al. (in preparation) (IRAC $3.6 \mu \mathrm{m}, 4.5 \mu \mathrm{m}$, $5.8 \mu \mathrm{m}, 8.0 \mu \mathrm{m}$ and MIPS $24 \mu \mathrm{m}$ ) and Elbaz et al. (2011) 

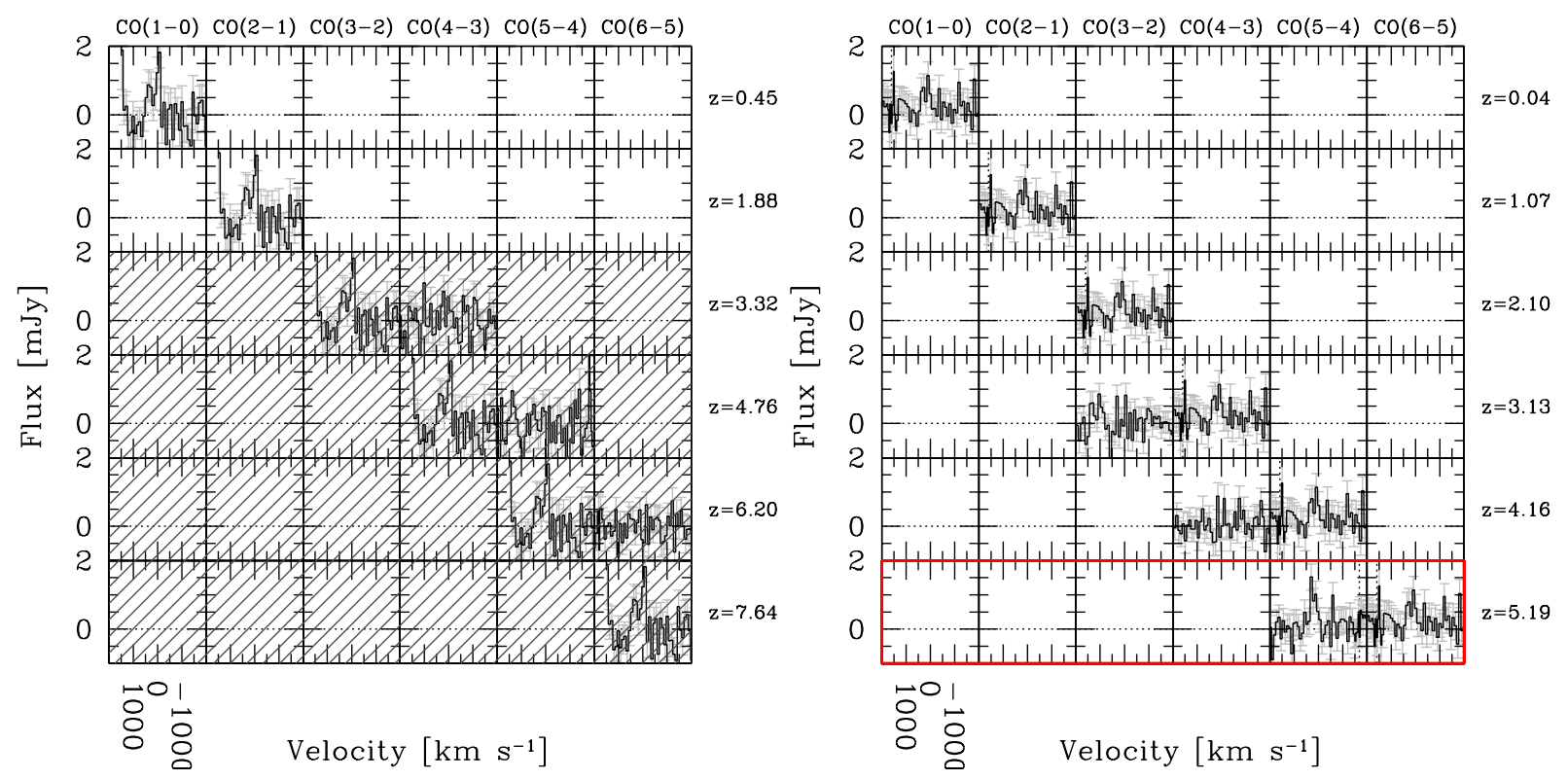

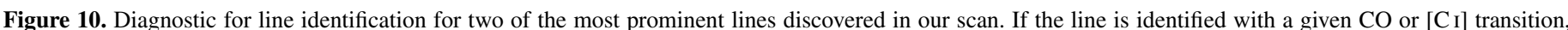

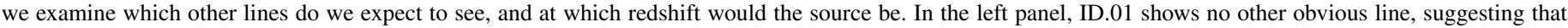

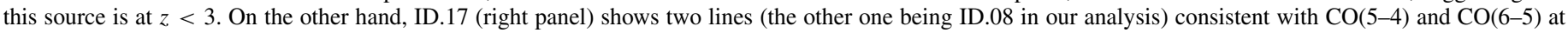
$z \approx 5.19$ (Walter et al. 2012).

(A color version of this figure is available in the online journal.)

(Herschel/PACS $100 \mu \mathrm{m}, 160 \mu \mathrm{m}$ and SPIRE $250 \mu \mathrm{m}, 350 \mu \mathrm{m}$, $500 \mu \mathrm{m}$ ). We also include limits on the $3 \mathrm{~mm}$ continuum emission, as derived from the map presented in Section 6. We fit these SEDs using magphys (da Cunha et al. 2008, 2013b). This physically motivated model combines the attenuated stellar emission from the UV to the NIR with the dust emission in the MIR to submillimeter assuming energy balance between the radiation absorbed at UV and optical wavelengths and the one re-radiated by dust at IR wavelengths. We shift galaxy templates to the redshifts corresponding to possible $\mathrm{CO}$ identifications of our line candidates. The available photometric information on the optical/NIR counterparts allow us to exclude some possible CO identifications. The potential counterparts of our CO line candidates have modest stellar masses: $M_{*} \lesssim 10^{10} M_{\odot}$. The only exception is the counterpart of ID.03 $\left(M_{*}=1.3 \times 10^{11} M_{\odot}\right.$, see discussion below).

\subsection{Notes on Individual Line Candidates}

Hereafter we briefly describe all the positive line candidates in our analysis. We divide the sample in "secure line detections," i.e., line candidates which have been confirmed through corollary observations; "high-quality line candidates," i.e., prominent line candidates which show a spectroscopic $\mathrm{S} / \mathrm{N}>3.5$ (labeled as quality 1 in Table 2); and others.

\subsubsection{Secure Line Detections}

ID.03 $(v=82.80 \pm 0.02 \mathrm{GHz})$ is the brightest line detected in our $3 \mathrm{~mm}$ scan. Our PdBI follow-up $2 \mathrm{~mm}$ observations reveal a second line at $\approx 165.5 \mathrm{GHz}$ (Figure 11). A third, tentative line is reported at $\approx 41.4 \mathrm{GHz}$ in our JVLA observations, albeit only at a $\mathrm{S} / \mathrm{N}$ of $\sim 3.6 \sigma$. These lines are consistent with being $\mathrm{CO}(2-1), \mathrm{CO}(4-3)$ and $\mathrm{CO}(1-0)$ at $z=1.784 .{ }^{21}$ The

\footnotetext{
${ }^{21}$ Given the relatively low $\mathrm{S} / \mathrm{N}$ of our detections, the detected lines could in principle also be the $\mathrm{CO}(4-3), \mathrm{CO}(8-7)$ and $\mathrm{CO}(2-1)$ transitions, which would imply a redshift of $z \sim 4.5$. However in this case the $\mathrm{CO}(5-4)$ line would also be covered by our $3 \mathrm{~mm}$ scan, but is not detected. This leaves $z=1.784$ as the only plausible redshift.
}

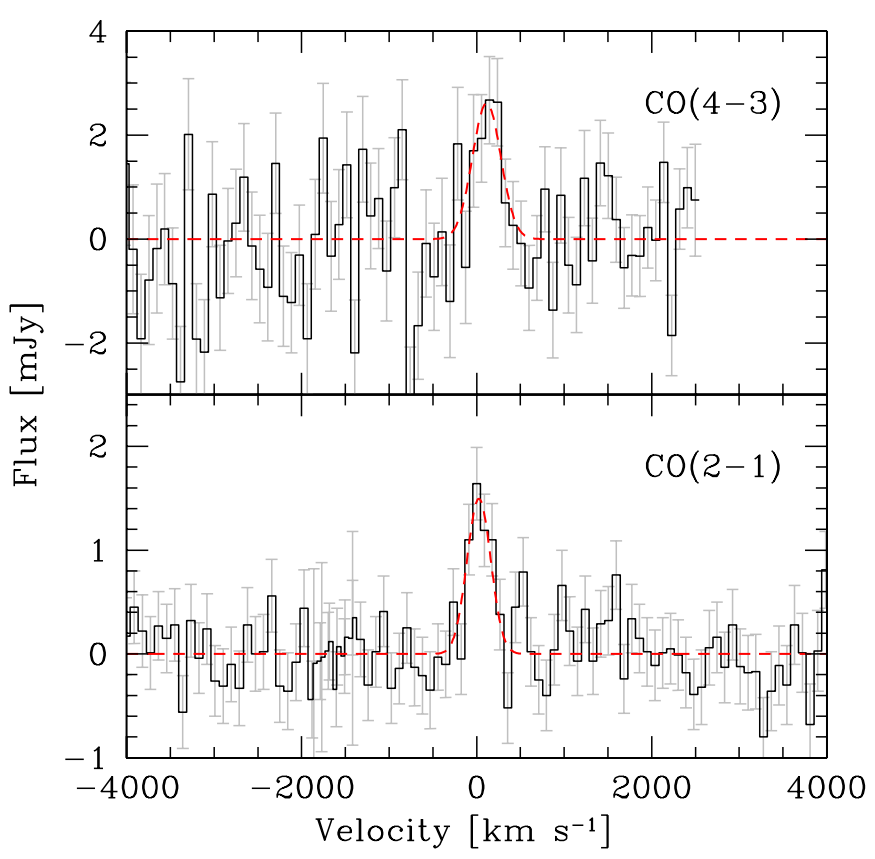

Figure 11. $\mathrm{CO}$ emission of $\mathrm{CO}(2-1)$ and $\mathrm{CO}(4-3)$ of ID.03 at $\mathrm{z}=1.784$ from our $3 \mathrm{~mm}$ and $2 \mathrm{~mm}$ PdBI observations. A Gaussian fit gives a peak line flux of $\sim 1.5 \mathrm{mJy}$ and $\sim 2.5$ for $\mathrm{CO}(2-1)$ and $\mathrm{CO}(4-3)$ respectively. The integrated line fluxes are $0.51 \pm 0.08 \mathrm{Jy} \mathrm{km} \mathrm{s}^{-1}$ and $1.06 \pm 0.23 \mathrm{Jy} \mathrm{km} \mathrm{s}^{-1}$. The implied luminosities are $L^{\prime}=2.0 \times 10^{10} \mathrm{~K} \mathrm{~km} \mathrm{~s}^{-1} \mathrm{pc}^{2}$ and $L^{\prime}=1.1 \times 10^{10} \mathrm{~K} \mathrm{~km} \mathrm{~s}^{-1} \mathrm{pc}^{2}$ respectively.

(A color version of this figure is available in the online journal.)

line fluxes are $\sim 0.11 \mathrm{Jy} \mathrm{km} \mathrm{s}^{-1}, 0.50 \pm 0.08 \mathrm{Jy} \mathrm{km} \mathrm{s}^{-1}$, and $1.06 \pm 0.23 \mathrm{Jy} \mathrm{km} \mathrm{s}^{-1}$ for $\mathrm{CO}(1-0), \mathrm{CO}(2-1)$, and $\mathrm{CO}(4-3)$, respectively. The $\mathrm{CO}(4-3) / \mathrm{CO}(2-1)$ flux ratio is $\approx 2$, i.e., close to the average CO ladder observed in SMGs (Carilli \& Walter 2013) and significantly higher than what is inferred from the (few) observations of multiple $\mathrm{CO}$ transitions in $\mathrm{Bz} K$ galaxies (Dannerbauer et al. 2009; Aravena et al. 2010). The observed 


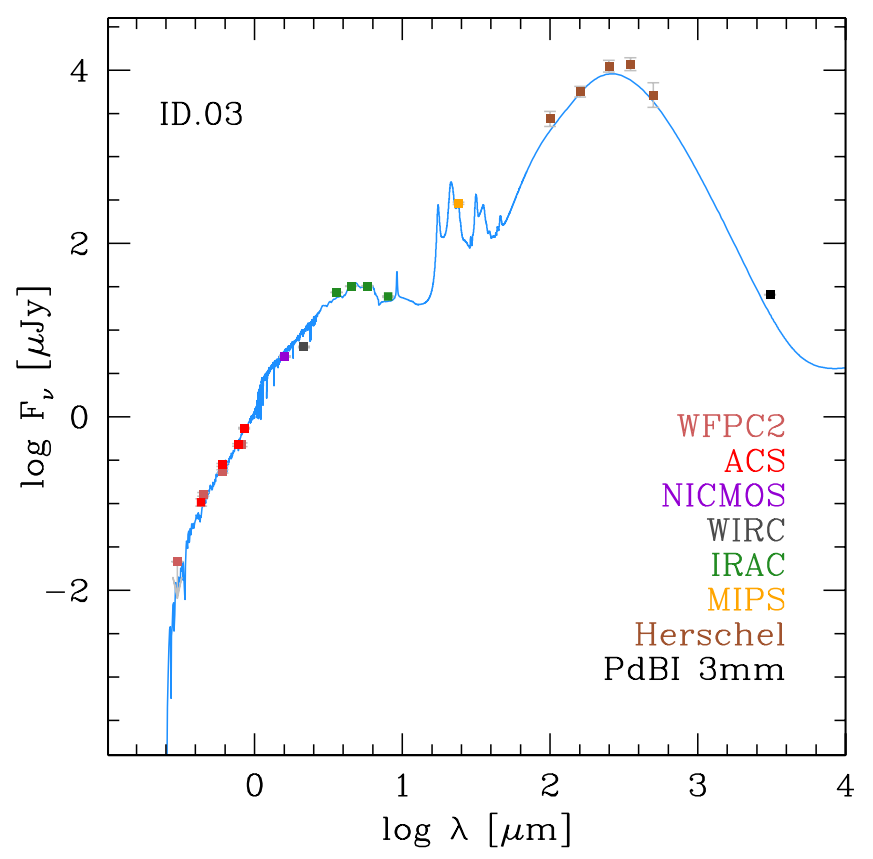

Figure 12. Spectral energy distribution of ID.03. Observed data are taken from: Williams et al. (1996) (WFPC2); Bundy et al. (2009) (ACS+WIRC); Dickinson et al. (2003) (NICMOS); M. Dickinson et al., in preparation (IRAC+MIPS); Elbaz et al. (2011) (Herschel) and this work (3 mm continuum). The fit (shown as a solid line) is performed following da Cunha et al. (2013b), assuming a redshift of $z=1.784$, as measured based on our $\mathrm{CO}(2-1)$ and $\mathrm{CO}(4-3)$ detections. In particular, the model of dust emission well fits the observed IR photometry.

(A color version of this figure is available in the online journal.)

$\mathrm{CO}(1-0)$ flux is also consistent with an SMG-like CO ladder, within the uncertainties. We therefore adopt a line luminosity $L_{\mathrm{CO}(1-0)}^{\prime}=2.4 \times 10^{10} \mathrm{~K} \mathrm{~km} \mathrm{~s}^{-1} \mathrm{pc}^{2}$, based not on our quite uncertain measurement of the $\mathrm{CO}(1-0)$ line, but on the $\mathrm{CO}(2-1)$ luminosity, assuming an $L_{\mathrm{CO}(2-1)}^{\prime} / L_{\mathrm{CO}(1-0)}^{\prime}$ ratio of 0.85 (Carilli \& Walter 2013).

ID.03 is coincident with an optically faint but infrared bright galaxy. Its $\mathrm{AB}$ magnitudes are: $B=26.4, z=24.2, K=21.9$ and thus $B z K=(z-K)-(B-z)=0.2$ (i.e., $B z K>-0.2$, the definition of a $B z K$ galaxy following Daddi et al. 2004). With $(B-z)_{\mathrm{AB}}=2.1$ and $(z-K)_{\mathrm{AB}}=2.3$ this galaxy is also placed inside the box of "star-forming galaxies at $z>1.4$ " (Figure 3 in Daddi et al. 2004). The galaxy is also detected by Herschel, sampling most of the dust continuum emission (Elbaz et al. 2011). This galaxy has no unambiguous spectroscopic redshift, although it has frequently been observed with Keck optical spectrographs. ${ }^{22}$ The WFPC $2+\mathrm{NICMOS}+\mathrm{K}$ photometric redshift for this galaxy is $z_{\text {phot }} \sim 1.6$, suggestively close to the $\mathrm{CO}$ redshift $z=1.784$. As shown in Figure 12, our $\mathrm{CO}$ redshift is in excellent agreement with the observed SED, ranging from the optical HST to Herschel photometry. The source has only a faint VLA $1.4 \mathrm{GHz}$ detection but is not detected by Chandra and is thus not known to host an AGN.

Based on the SED fitting we derive the following parameters of the galaxy (median-likelihood estimates; $1 \sigma$ confidence ranges in brackets): $s$ tar formation rate $(\mathrm{SFR})=38_{-1}^{+8} M_{\odot} \mathrm{yr}^{-1}$; $M_{*}=\left(2.5_{-0.3}^{+0.1}\right) \times 10^{11} M_{\odot} ; L_{\text {dust }}=\left(6.8_{-0.16}^{+0.65}\right) \times 10^{11} L_{\odot}$; $\mathrm{M}_{\text {dust }}=\left(4.6_{-3.0}^{+10}\right) \times 10^{7} M_{\odot}$. Figure 13 shows the location

\footnotetext{
22 NIR continuum emission is detected in HST/WFC3 grism observations covering 1.1-1.65 $\mu \mathrm{m}$ from A Grism $\mathrm{H}$-alpha SpecTroscopy survey (AGHAST; B. Weiner et al., in preparation). No strong emission line, in particular the [O III] emission, is seen, implying that the line is either intrinsically weak or absorbed by dust.
}

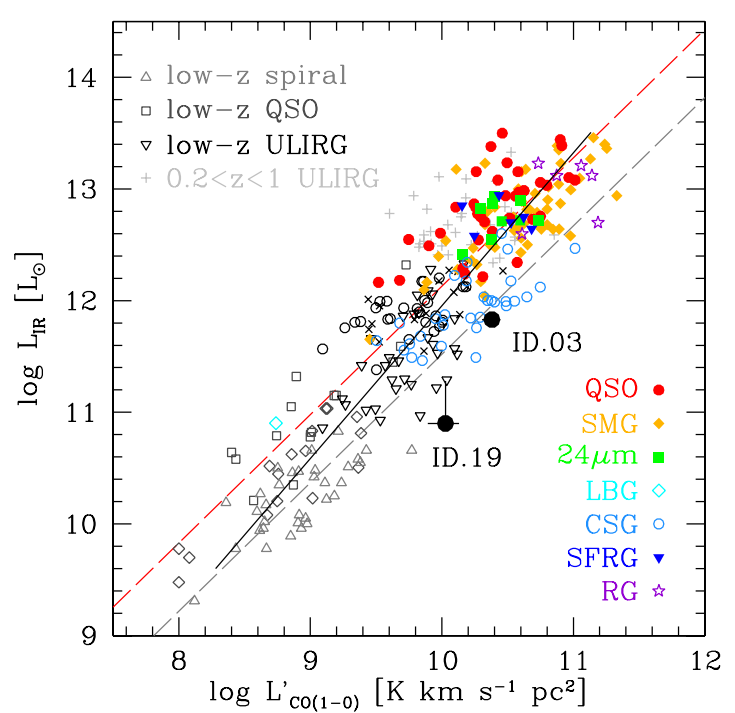

Figure 13. IR luminosity as a function of $\mathrm{CO}(1-0)$ luminosity of the two secure line candidates discovered in our survey, beside HDF 850.1. For ID.03, we use the $\mathrm{CO}(1-0)$ luminosity extrapolated from the $\mathrm{CO}(2-1)$ and assuming a typical SMG CO excitation curve (see text for details). For ID.19, we infer CO(1-0) based on the $\mathrm{CO}(3-2)$ luminosity, assuming M82-like excitation. The large uncertainties in IR luminosity for this source are due to the lack of photometric constraints a wavelengths longer than $24 \mu \mathrm{m}$ (see Figure 15). For a comparison, we plot in rainbow-colored symbols different classes of galaxies at $z>1$, and with gray symbols galaxies at $z<1$, from the compilation by Carilli \& Walter (2013). The two sources discussed in our study both lie below the typical IR-CO(1-0) luminosity relation, but still within the observed scatter, once we account for observational uncertainties.

(A color version of this figure is available in the online journal.)

of ID.03 with respect to the $L_{\mathrm{IR}}-L_{\mathrm{CO}(1-0)}^{\prime}$ relation (Carilli \& Walter 2013). ID.03 is found within the observed scatter of the relation, in the region populated by color-selected galaxies at $z>1$, and about one order of magnitude fainter (in terms of IR luminosity) than typical SMGs. The galaxy is quite massive and has a specific star formation rate of sSFR $\sim 0.15 \pm 0.04 \mathrm{Gyr}^{-1}$ and is about one order of magnitude below the sSFR of an average "main-sequence" galaxy at $z \sim 1.7$ (e.g., Elbaz et al. 2011). Given its molecular gas mass of $\sim(9 \pm 2) \times 10^{10} M_{\odot}$ (obtained by assuming $\alpha_{\mathrm{CO}}=3.6 M_{\odot}\left(\mathrm{K} \mathrm{km} \mathrm{s}^{-1} \mathrm{pc}^{2}\right)^{-1}$; see Daddi et al. 2010), we derive a star formation efficiency of SFR/ $M_{\mathrm{H} 2} \sim 0.4 \mathrm{Gyr}^{-1}$, or a gas depletion time of order $\sim 2 \mathrm{Gyr}$.

ID.08 ( $v=93.17 \pm 0.03 \mathrm{GHz})$ and its companion ID. 17 are both spatially consistent with the SMG HDF 850.1. The two lines are identified as $\mathrm{CO}(5-4)$ and $\mathrm{CO}(6-5)$ at $z=5.183$. In the Appendix we present additional observations of this source, encompassing the $\mathrm{CO}(7-6)$ and $[\mathrm{C}]_{2-1}$ lines. We refer to Walter et al. (2012) for more details on this galaxy.

ID.17 ( $v=111.85 \pm 0.06 \mathrm{GHz}$ ), together with ID.08 (see above), pins down the redshift of the SMG HDF 850.1 at $z=5.183$.

ID.19 (113.45 $\pm 0.04 \mathrm{GHz})$ is associated with a galaxy at R.A. $=12: 36: 51.61$, decl. $=+62: 12: 17.3$, for which grism spectroscopic redshift $z_{\text {grism }}=2.044 \pm 0.002$ is available through AGHAST (B. Weiner et al., in preparation; see Figure 14). This redshift is in excellent agreement with the redshift inferred from the CO line detection in our study $(z=2.0474 \pm 0.0015)$, under the assumption that the line is identified with $\mathrm{CO}(3-2)$. The available photometry of this source samples a broad range of wavelengths, up to MIPS $24 \mu \mathrm{m}$. The source is formally not detected in Herschel maps, although a bright, nearby foreground $(z=0.300)$ galaxy prevents us from directly measuring the FIR emission in the counterpart of ID.19. From the SED fit 

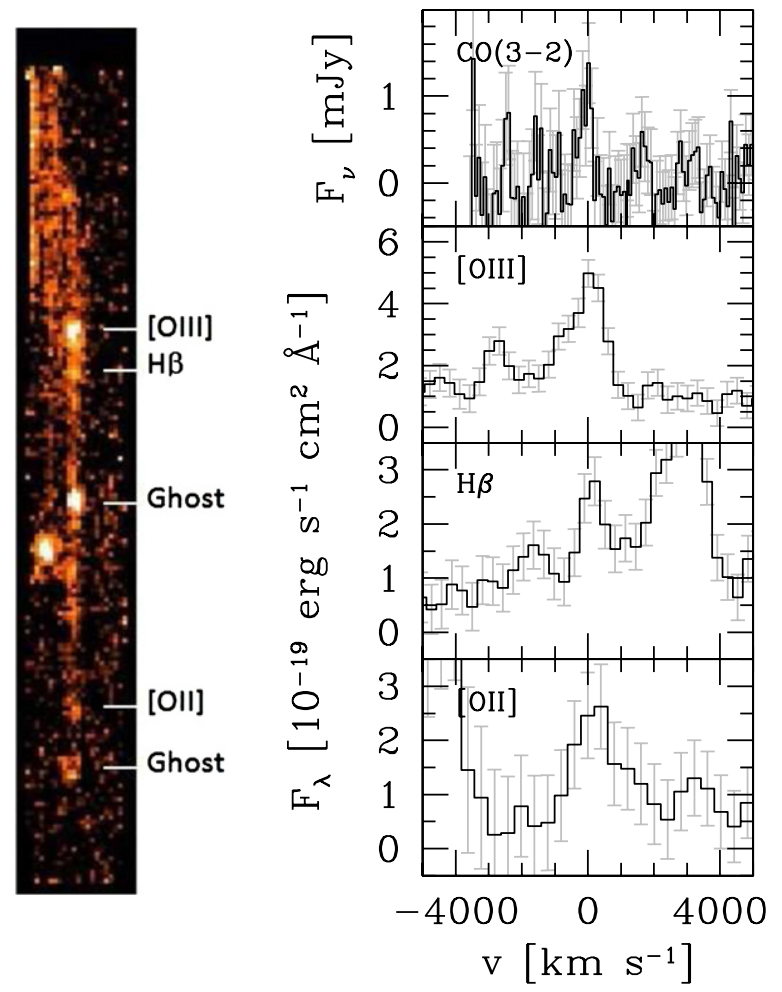

Figure 14. Left: near-infrared, two-dimensional grism spectrum of the counterpart associated with the line candidate ID.19, observed with HST as part of the AGHAST survey (B. Weiner et al., in preparation). Wavelength increases from bottom to the top. Main emission lines, identified assuming $z=2.047$, and ghost images from the zero order continuum emission are labeled. Right: velocity profile of the $\mathrm{CO}(3-2)$ line of ID.19 from our $3 \mathrm{~mm}$ observations (top), and of the rest-frame optical lines shown in the left hand panel. There is excellent agreement in redshift between those lines. We note that rest-frame optical lines appear broadened because of the modest spectral resolution of the grism observations.

(A color version of this figure is available in the online journal.)

(Figure 15), we infer the following properties of the counterpart of ID.19: $\mathrm{SFR}=7.9_{-1.4}^{+3.5} M_{\odot} \mathrm{yr}^{-1} ; M_{*}=(1.9 \pm 0.3) \times 10^{10} M_{\odot}$; $L_{\text {dust }}=\left(8_{-1}^{+11}\right) \times 10^{10} L_{\odot} ; M_{\text {dust }}=\left(9_{-7}^{+50}\right) \times 10^{6} M_{\odot}$. The galaxy is thus quite massive and has a specific star formation rate of sSFR $\approx 0.4 \mathrm{Gyr}^{-1}$, below the sSFR of an average "mainsequence" galaxy at $z \sim 2.05$ (e.g., Elbaz et al. 2011). We measure a line luminosity $L_{\mathrm{CO}(3-2)}^{\prime}=(9.9 \pm 2.6) \times 10^{9} \mathrm{~K} \mathrm{~km} \mathrm{~s}^{-1} \mathrm{pc}^{2}$. Assuming a Milky-Way-like (MW-like) CO excitation, the corresponding $\mathrm{CO}(1-0)$ luminosity would be $L_{\mathrm{CO}(1-0)}^{\prime} \approx 3.7 \times$ $10^{10} \mathrm{~K} \mathrm{~km} \mathrm{~s}^{-1} \mathrm{pc}^{2}$. We note that this value is significantly larger than what is observed in local spiral galaxies of comparable IR luminosity. The discrepancy might be reduced by a factor of 3.5 if the true $\mathrm{CO}$ excitation is higher than the MW-like excitation that we assume. By adopting an M82like $\mathrm{CO}$ excitation (Weiß et al. 2007), we obtain $L_{\mathrm{CO}(1-0)}^{\prime} \approx$ $1.1 \times 10^{10} \mathrm{~K} \mathrm{~km} \mathrm{~s}^{-1} \mathrm{pc}^{2}$, which locates ID. 19 within the scatter of the observed $L_{\mathrm{IR}}-L_{\mathrm{CO}(1-0)}^{\prime}$ relation (see Figure 13), among local ultra-luminous IR galaxies. Assuming $\alpha_{\mathrm{CO}}=3.6 M_{\odot} /(\mathrm{K}$ $\left.\mathrm{km} \mathrm{s}^{-1} \mathrm{pc}^{2}\right)^{-1}$, following Daddi et al. (2010), we derive the total molecular gas mass $M_{\mathrm{H} 2} \sim 1.3 \times 10^{11} M_{\odot}$, although this estimate is significantly depends on the $\mathrm{CO}$ excitation and on the choice of $\alpha_{\mathrm{CO}}$.

\subsubsection{High-quality Line Candidates}

ID.01 $(v=80.05 \pm 0.03 \mathrm{GHz})$ is a prominent line candidate (the third most significant line, in terms of $\mathrm{S} / \mathrm{N}_{\mathrm{spec}}$ ).

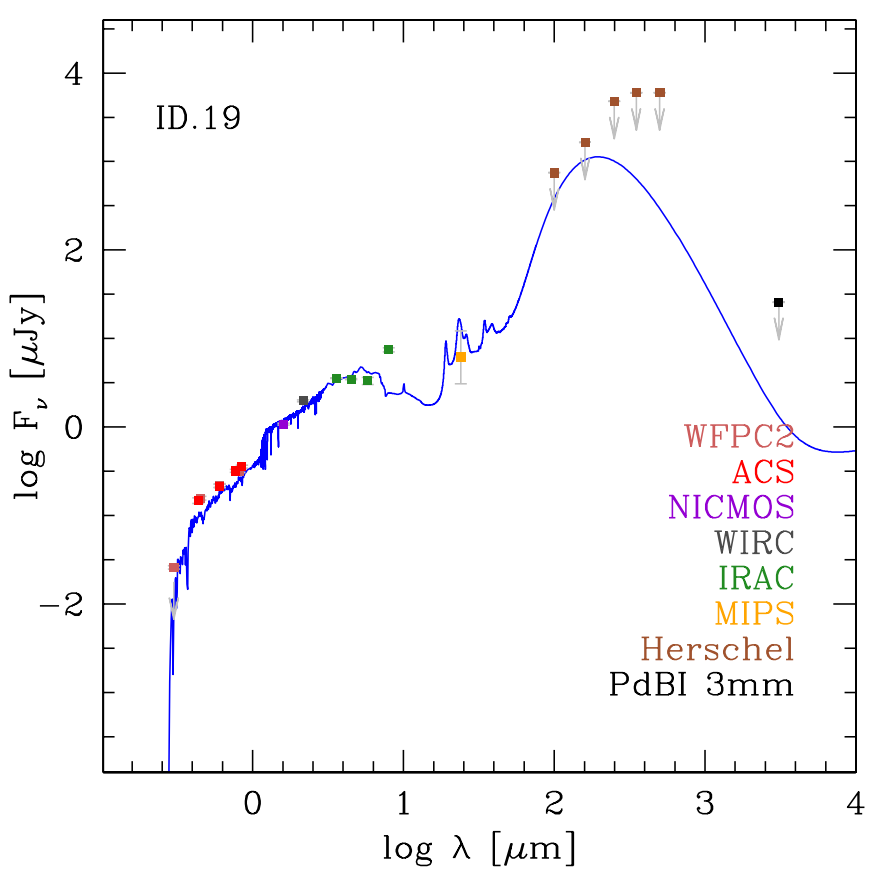

Figure 15. Spectral energy distribution of ID.19. Symbol code is the same as in Figure 12. The fit (shown as a solid line) assumes a redshift of $z=2.047$, based on our $\mathrm{CO}(3-2)$ detection (see Figure 14).

(A color version of this figure is available in the online journal.)

The line profile may be indicative of two peaks separated by $\sim 300 \mathrm{~km} \mathrm{~s}^{-1}$, but the $\mathrm{S} / \mathrm{N}$ is too low for a proper line profile characterization. The lack of a second line at the same position suggests that the source is at $z=0.440$ (if the line were identified as $\mathrm{CO}(1-0)$ ) or $z=1.8799$ (if the line were $\mathrm{CO}(2-1)$ ). The source has no optical/NIR counterpart, which makes the lower- $z$ interpretation unlikely.

ID.02 ( $v=82.07 \pm 0.02 \mathrm{GHz})$ is at the edge of the primary beam area in one of the lowest frequency setups in the scan. It has two possible counterparts (see Figure 16), with $z_{\text {phot }}=0.92$ and 1.12 in Fernández-Soto et al. (1999). The line can be interpreted as $\mathrm{CO}(1-0)$ at $z=0.41$ or as $\mathrm{CO}(2-1)$ at $z=1.82$. The SED fit favors the latter scenario for both counterparts. The first of the two galaxies seems to be the most plausible counterpart, since it is best fitted assuming higher dust extinction in the optical bands than in the companion, implying brighter FIR emission (and therefore, likely brighter $\mathrm{CO}$ fluxes). An alternative scenario to explain the relatively blue color of the optical counterparts and the bright $\mathrm{CO}$ emission in this source (and possibly in other cases in our sample) is that the optical counterparts are acting as gravitational lenses of a background, CO-bright source. We note however that the lensing hypothesis is unlikely affecting more than a few sources in our sample (if any).

ID.05 $(v=89.89 \pm 0.04 \mathrm{GHz})$ shows no second line at the same spatial position, suggesting $z_{\mathrm{CO}}<3.016$. The lack of any optical/NIR counterpart makes an identification as $\mathrm{CO}(1-0)\left(z_{\mathrm{CO}}=0.2824\right)$ unlikely. The two remaining options are $\mathrm{CO}(2-1)$ at $z=1.5648$ or $\mathrm{CO}(3-2)$ at $z=2.8471$. The latter is preferred due to the lack of any optical counterparts.

ID.10 ( $v=103.88 \pm 0.03 \mathrm{GHz})$ can be identified as $\mathrm{CO}(2-1)$ at $z=1.219$ or $\mathrm{CO}(3-2)$ at $z=2.329$. In our analysis, we adopt the latter (based on the lack of optical/NIR counterparts). We note however that a few other galaxies with optical/NIR spectroscopic redshift $z \approx 1.2$ are observed in the field, tentatively pointing toward a galaxy overdensity at this redshift. 


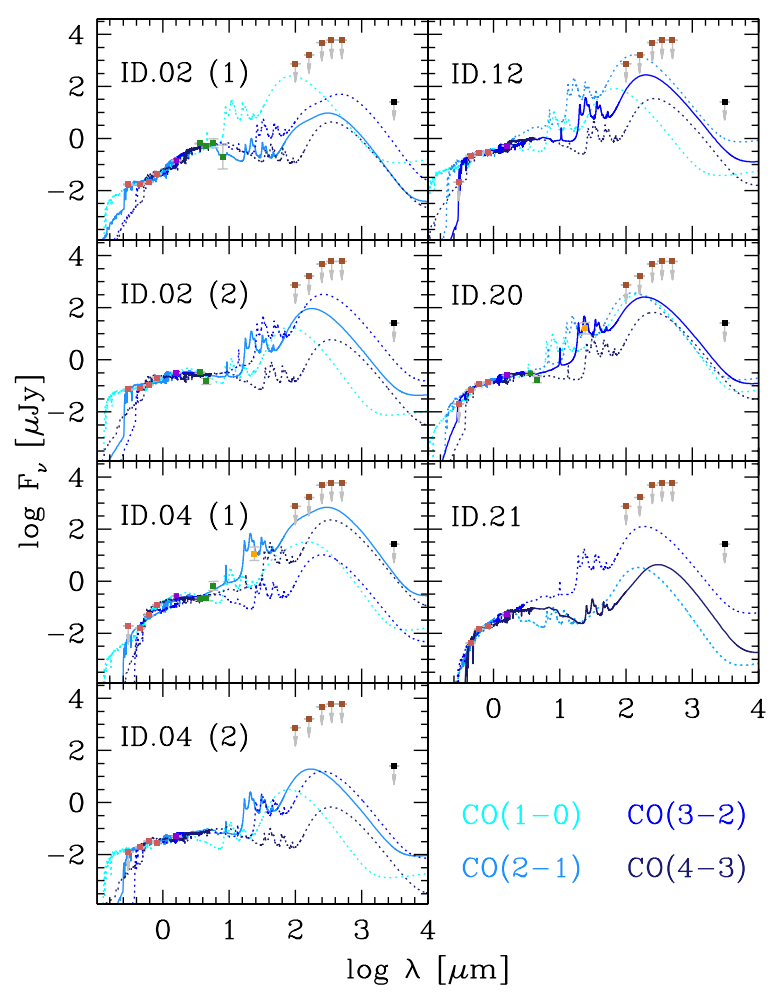

Figure 16. Observed spectral energy distributions of the optical/NIR counterparts of other (positive) CO line candidates of our molecular line scan. Symbols are coded as in Figure 12. Upper limits $(3 \sigma)$ are plotted as downward arrows. For each galaxy, we fit the observed photometry assuming four different redshifts, each associated with a different possible identification of the $\mathrm{CO}$ line candidate discovered in our scan (from cyan to dark blue: $\mathrm{CO}(1-0), \mathrm{CO}(2-1), \mathrm{CO}(3-2)$ and $\mathrm{CO}(4-3))$. Fits that are associated with our adopted $z_{\mathrm{CO}}$ are highlighted with solid lines. For ID.02 and ID.04, two possible counterparts are identified (see Table 3).

(A color version of this figure is available in the online journal.)

If that was indeed the case, the redshift assignment for ID.10 should be re-considered.

ID.11 ( $v=108.32 \pm 0.03 \mathrm{GHz})$ has no optical/NIR counterparts. Plausible interpretations are therefore $\mathrm{CO}(2-1)$ at $z=1.128$ or $\operatorname{CO}(3-2)$ at $z=2.192$ and we adopt the latter redshift, given the absence of emission at optical/NIR wavelengths.

ID.12 ( $v=108.43 \pm 0.04 \mathrm{GHz})$ is spatially consistent with a pair of galaxies detected at optical/NIR wavelengths. One of them has a redshift $z=0.9614$ (Barger et al. 2008), which is not consistent with any $\mathrm{CO}$ identification of the line detected in our $3 \mathrm{~mm}$ scan. On the other hand, the second galaxy has a photometric redshift of $z=2.08$ (Fernández-Soto et al. 1999), consistent with the identification of this line as $\mathrm{CO}(3-2)$ at $z=2.1891$. The SED of this galaxy suggests that this is a rather blue galaxy with $L_{\text {dust }} \approx 8 \times 10^{9} L_{\odot}$. The Herschel/PACS $100 \mu \mathrm{m}$ observation of the field reveals a tentative detection ( $\sim 1 \mathrm{mJy}$, close to the $3 \sigma$ sensitivity limit) spatially consistent with this source. Such a flux is significantly brighter than what is predicted in our SED fits if that data point is ignored (see Figure 16). Since the $100 \mu \mathrm{m}$ emission is partially blended with the much brighter $B z K$ counterpart of ID.03, and possibly with other sources, we opt to treat this measurement as an upper limit.

ID.15 $(v=110.55 \pm 0.04 \mathrm{GHz})$ has no counterpart (the closest galaxy in the $H S T$ /NICMOS $H$-band observations is $>3^{\prime \prime}$ away). We identify the line as $\mathrm{CO}(3-2)$ at $z=2.150$. Alternatively, the line could be $\mathrm{CO}(2-1)$ at $z=1.100$.
ID.18 ( $v=112.63 \pm 0.04 \mathrm{GHz})$ is the second most significant line detection in our survey. We verified that the line is likely real by splitting the data taken in this frequency setup into four independent blocks, and searching for the line in each of them. This test retrieves the line at the expected significance in each setup. The probability of this line being a real astrophysical source is $\sim 95 \%$ in the analysis by L. Lentati et al. (in preparation). The line flux is $0.58 \pm 0.11 \mathrm{Jy} \mathrm{km} \mathrm{s}^{-1}$. No other line is reported in the $3 \mathrm{~mm}$ scan at this spatial position. This excludes a high- $J$ identification. On the other hand, no galaxy counterpart is observed at any optical/NIR/MIR wavelengths. The lack of counterparts likely rules out a low- $z$ interpretation (excluding anything below $z \sim 1.5$, down to stellar luminosities comparable with the Large Magellanic Cloud). This suggests that the line is most likely $\mathrm{CO}(3-2)$ at $z=2.071$. As in all previous cases, a measurement of a second $\mathrm{CO}$ line is required to confirm this redshift identification.

ID.20 (113.45 $\pm 0.05 \mathrm{GHz})$ is associated with a galaxy at R.A. $=12: 36: 50.396$, decl. $=+62: 12: 05.12$. Our CO observations suggest that the source is $\mathrm{CO}(3-2)$ at $z=2.05$, a redshift identification that is supported by the observed photometry (see Figure 16). Our SED fit yields a stellar mass $M_{*}=(1.21 \pm 0.07) \times 10^{9} M_{\odot}$, a dust luminosity of $L_{\text {dust }}=(5.6 \pm 1.2) \times 10^{10} L_{\odot}$, and an associated SFR $=$ $1.0 \pm 0.3 M_{\odot} \mathrm{yr}^{-1}$. The $\mathrm{CO}(3-2)$ line luminosity is $L_{\mathrm{CO}(3-2)}^{\prime}=$ $(8.8 \pm 2.5) \times 10^{9} \mathrm{~K} \mathrm{~km} \mathrm{~s}^{-1} \mathrm{pc}^{2}$. Under the same assumptions used for ID.19, we derive $L_{\mathrm{CO}(1-0)}^{\prime} \approx 3.2 \times 10^{10} \mathrm{~K} \mathrm{~km} \mathrm{~s}^{-1} \mathrm{pc}^{2}$.

\subsubsection{Other Line Candidates}

ID.04 $(84.94 \pm 0.03 \mathrm{GHz})$ is a low-quality line candidate in our search. It can be interpreted as $\mathrm{CO}(1-0)$ at $z=0.36$ or $\mathrm{CO}(2-1)$ at $z=1.72$. It has two possible counterparts (see Table 3 and Figure 16). The redshift $z=1.72$ hypothesis for the second galaxy yields $L_{\mathrm{IR}} \sim 7 \times 10^{10} L_{\odot}$, i.e., more than one order of magnitude brighter IR emission than the low-redshift case. We conclude that a $\mathrm{CO}$ redshift at $z=1.72$ is more plausible.

ID.13 $(v=108.77 \pm 0.03 \mathrm{GHz})$ is a low-quality line candidate without optical/NIR counterparts. If real, plausible interpretations are $\mathrm{CO}(2-1)$ at $z=1.120$ or, most likely, $\mathrm{CO}(3-2)$ at $z=2.179$.

ID.14 ( $v=109.65 \pm 0.03 \mathrm{GHz})$ lacks optical/NIR counterparts. The line is flagged as low-quality in our analysis. Plausible interpretations are $\mathrm{CO}(2-1)$ and $\mathrm{CO}(3-2)$ at $z=1.102$ and $z=2.154$, respectively.

ID.21 (114.15 $\pm 0.05 \mathrm{GHz})$ is a low-quality line candidate, tentatively associated with a faint galaxy at R.A. = 12:36:51.358, decl. $=+62: 12: 11.89$ (see Figure 16). Given the faintness of the source and its colors, a relatively high- $z$ scenario $(z \sim 2-3)$ is favored. The lack of a second $\mathrm{CO}$ detection in our scan would point toward the $z=2.03$ scenario, although the signal-to-noise of our CO detection is modest, and we cannot rule out the $z=3.04$ case, which is also acceptable given the available optical/NIR photometry.

\section{COMPARISON WITH EXPECTATIONS}

In the following analysis, we only consider the "secure" and "high-quality" line candidates.

In Figure 17 we compare the total number of line candidates in our scan with empirical predictions. From the list of all the positive line candidates in Table 2, we have omitted the line candidates ID.08 and ID.17, which are associated with 
Table 3

Identification of the Line Candidates in Our Analysis and Tentative Optical/NIR Counterparts

\begin{tabular}{|c|c|c|c|c|c|c|c|c|c|}
\hline (1) & $\begin{array}{l}\text { Counter- } \\
\text { part? } \\
(2)\end{array}$ & (3) & (4) & $\begin{array}{l}\text { Dist. } \\
\left({ }^{\prime \prime}\right) \\
(5)\end{array}$ & $\begin{array}{l}z_{\text {phot }} \\
(6)\end{array}$ & $\begin{array}{l}J_{\text {up }} \\
(7)\end{array}$ & $\begin{array}{l}z \mathrm{CO} \\
(8)\end{array}$ & $\begin{array}{c}L^{\prime} \\
\left(10^{9} \mathrm{~K} \mathrm{~km} \mathrm{~s}^{-1} \mathrm{pc}^{2}\right) \\
(9)\end{array}$ & $\begin{array}{c}L_{\mathrm{c}} \\
\left(10^{6} L_{\odot}\right) \\
(10)\end{array}$ \\
\hline ID.01 & $\mathrm{N}$ & & & & & $(1), 2$ & $1.8799 \pm 0.0012$ & 23.4 & 9.2 \\
\hline ID.02 (1) & $\mathrm{Y}$ & $12: 36: 52.245$ & $+62: 12: 51.56$ & 1.3 & 0.92 & $(1), 2$ & $1.8084 \pm 0.0007$ & 15.3 & 6.0 \\
\hline ID.02 (2) & $\mathrm{Y}$ & $12: 36: 52.561$ & $+62: 12: 51.56$ & 1.5 & 1.12 & & & & \\
\hline ID.03 & $\mathrm{Y}$ & $12: 36: 48.626$ & $+62: 12: 15.79$ & 0.4 & 1.6 & 2 & $1.7844 \pm 0.0008$ & 20.1 & 7.9 \\
\hline ID.04 (1) & $\mathrm{Y}$ & $12: 36: 52.561$ & $+62: 12: 33.61$ & 1.5 & 0.64 & $(1), 2$ & $1.7142 \pm 0.0007$ & 7.0 & 2.8 \\
\hline ID.04 (2) & $\mathrm{Y}$ & $12: 36: 52.485$ & $+62: 12: 35.46$ & 0.9 & 2 & & & & \\
\hline ID.05 & $\mathrm{N}$ & & & & & $(2), 3$ & $2.8471 \pm 0.0012$ & 19.0 & 2.52 \\
\hline ID.08 & $\mathrm{N}$ & & & & & 5 & $5.1869 \pm 0.0035$ & 15.9 & 9.71 \\
\hline ID. 10 & $\mathrm{~N}$ & & & & & $(2), 3$ & $2.3286 \pm 0.0008$ & 5.4 & 7.1 \\
\hline ID.11 & $\mathrm{N}$ & & & & & $(2), 3$ & $2.1924 \pm 0.0008$ & 9.3 & 1.23 \\
\hline ID. 12 & $\mathrm{Y}$ & $12: 36: 49.673$ & $+62: 12: 19.69$ & 0.8 & 2.08 & 3 & $2.1891 \pm 0.0012$ & 6.7 & 8.9 \\
\hline ID. 13 & $\mathrm{~N}$ & & & & & $(2), 3$ & $2.1794 \pm 0.0010$ & 6.2 & 8.2 \\
\hline ID.14 & $\mathrm{N}$ & & & & & $(2), 3$ & $2.1535 \pm 0.0009$ & 6.1 & 8.0 \\
\hline ID. 15 & $\mathrm{~N}$ & & & & & $(2), 3$ & $2.1497 \pm 0.0009$ & 11.4 & 15.1 \\
\hline ID. 17 & $\mathrm{~N}$ & & & & & 6 & $5.1856 \pm 0.0034$ & 9.5 & 101.0 \\
\hline ID. 18 & $\mathrm{~N}$ & & & & & $(2), 3$ & $2.0702 \pm 0.0010$ & 13.6 & 18.0 \\
\hline ID.19 & $\mathrm{Y}$ & $12: 36: 51.602$ & $+62: 12: 17.28$ & 1.5 & 1.76 & 3 & $2.0474 \pm 0.0015$ & 9.9 & 13.1 \\
\hline ID. 20 & $\mathrm{Y}$ & $12: 36: 50.396$ & $+62: 12: 05.12$ & 1.0 & 2.56 & 3 & $2.0479 \pm 0.0007$ & 8.8 & 11.7 \\
\hline ID. 21 & $\mathrm{Y}$ & $12: 36: 51.358$ & $+62: 12: 11.89$ & 1.0 & 3.16 & (3), 4 & $3.0383 \pm 0.0021$ & 9.1 & 28.5 \\
\hline
\end{tabular}

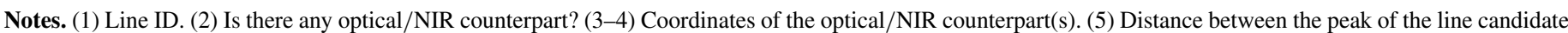

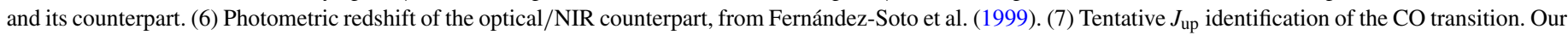

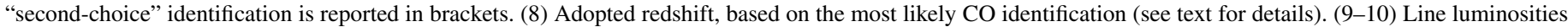
at the adopted $z_{\mathrm{CO}}$. The luminosity numbers are uncertain by a significant factor $(\sim 30 \%)$.

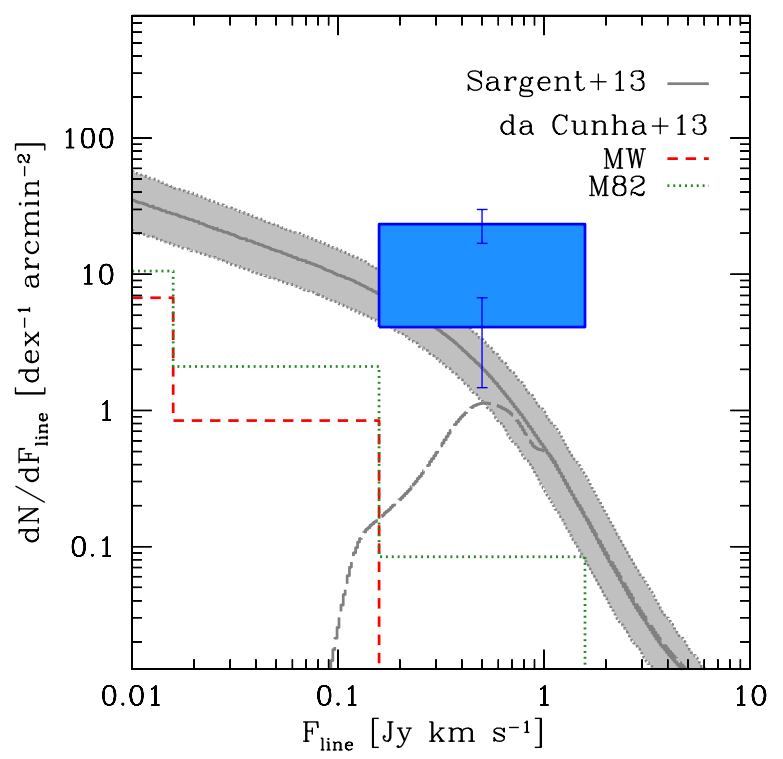

Figure 17. Comparison between the observed number of $\mathrm{CO}$ line candidates (shaded box), and the expectations from the empirical predictions presented in Sargent et al. (2013) and da Cunha et al. (2013b) (in the case of Milky Way-like $\mathrm{CO}$ excitation and in the case of M82-like excitation). The gray shading shows the $1 \sigma$ uncertainties in the predictions by Sargent et al. (2013). The long-dashed line shows the expected observed distribution, based on the predictions by Sargent et al. (2013) multiplied by the detection fraction discussed in Section 3.4. The shaded area spans a 1 dex CO flux bin (encompassing all the line candidates in our study). The bottom side is set by the two secure detections ID.03 and ID.19 (HDF 850.1 is not included here, since it is not representative of the general population of galaxies in the field). The upper side of the box is set by the total number of high-quality line candidates. In the area normalization, we took into account the actual beam size at the frequency of each line candidate. Error bars show Poissonian uncertainties in the observed line flux distribution. (A color version of this figure is available in the online journal.)
HDF 850.1, as they are not representative of the general field population of galaxies (our pointing center was chosen such to include HDF 850.1). We are therefore left with two secure line detections, plus nine high-quality (positive) line candidates. The contribution of each line is normalized by the actual size of the primary beam at the frequency of each line. All the lines are then grouped into a single, 1 dex wide flux bin.

We compare the observed flux distribution of our line candidates with the expected distributions of low- $J$ CO transitions based on the predictions by Sargent et al. $(2012,2013)$ and da Cunha et al. (2013b). Sargent et al. (2012, 2013) derived empirical predictions for the $\mathrm{CO}$ and IR luminosity functions of galaxies and for their evolution as a function of cosmic time. The framework is a description of star formation as a two-mode phenomenon. This divides galaxies into a "main-sequence" and a "starburst" population (see also Elbaz et al. 2011). The main driver of this distinction is the sSFR: star formation efficiencies for main-sequence galaxies are based on the observed Schmidt-Kennicutt law; starbursts deviate from this law due to an enhancement in their SFRs. The CO-to- $\mathrm{H}_{2}$ conversion factor $\alpha_{\mathrm{CO}}$ is assumed to depend on the metallicity (Z), scaling as $\sim Z^{-1}$ for main-sequence galaxies, while for starbursts $\alpha_{\mathrm{CO}}$ is strongly sensitive to the SFR-enhancement. Once folded into the evolution of the stellar mass function of star-forming galaxies and the evolution of the sSFR, this approach infers IR luminosity function of main-sequence and starbursting galaxies, and thus $\mathrm{CO}(1-0)$ luminosity functions. These are then expanded toward higher $J$ by assuming the $\mathrm{CO}$ excitation ladder of $B z K 21000$ (Dannerbauer et al. 2009; Daddi et al. 2010; Aravena et al. 2010; Casey et al. 2011) for main-sequence galaxies at $z>1$, and of GN20 (Daddi et al. 2009; Carilli et al. 2011; Bothwell et al. 2013) for starbursts. The resulting distributions are dominated by $J<4$ transitions for CO fluxes $\gtrsim 0.1 \mathrm{Jy} \mathrm{km} \mathrm{s}^{-1}$. 

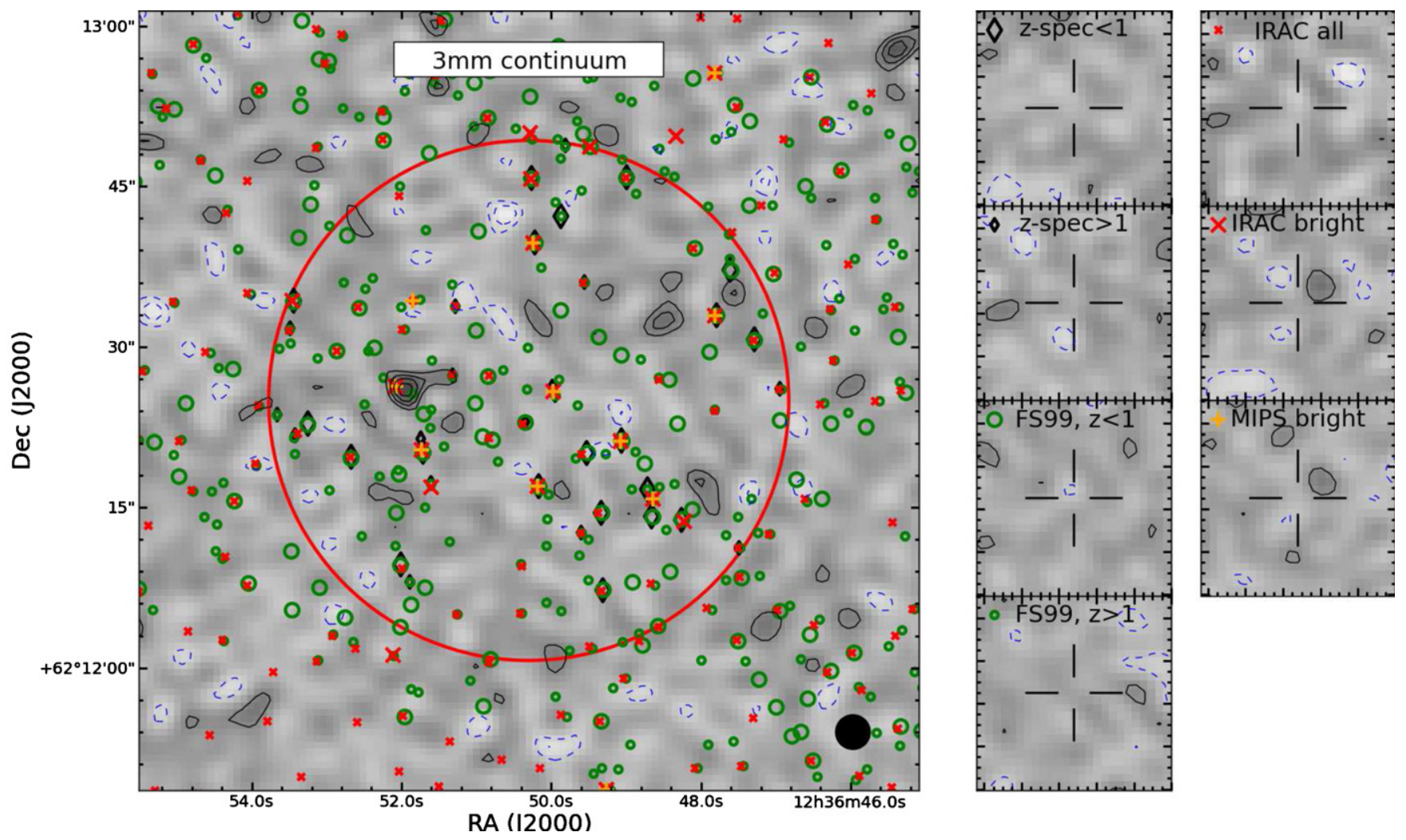

Figure 18. Left: $3 \mathrm{~mm}$ continuum image of the region of the HDF-N covered in our scan. Solid black/dashed blue contours show positive/negative $2,3,4, \ldots \sigma$ contours $\left(1 \sigma=8.6 \mu \mathrm{Jy}\right.$ beam $\left.^{-1}\right)$. The big solid red circle shows the FWHM of the primary beam size at $97.25 \mathrm{GHz}$ (i.e., the central frequency of our scan). Black diamonds mark the position of galaxies with spectroscopic redshifts (big/small symbols refer to objects at $z_{\text {spec }}>1 /<1$ ). Green circles label optically selected galaxies from Fernández-Soto et al. (1999) (FS99, bigger symbols highlight galaxies with photometric redshift $z_{\text {phot }}<1$ ). Red crosses mark IRAC-detected sources (bigger symbols identify the "IRAC-bright" sample, i.e., sources with IRAC $3.6 \mu \mathrm{m}$ fluxes brighter than $3.5 \mu \mathrm{Jy}$ ). Orange "+" symbols show MIPS-selected galaxies, i.e., sources with MIPS $24 \mu \mathrm{m}$ fluxes brighter than $20 \mu \mathrm{Jy}$. The beamsize of the observations is indicated in the bottom right corner. Right: for each subsample of galaxies, we show the postage stamp of the stacked emission. Each panel is $18^{\prime \prime} \times 18^{\prime \prime}$ wide (i.e., same scale as in the main panel). Only sources within the primary beam are considered in the stacking analysis. No detection is reported in any of the samples down to stacked continuum limits of only a few $\mu \mathrm{Jy}$ (Table 5).

(A color version of this figure is available in the online journal.)

da Cunha et al. (2013b), on the other hand, fitted the SED of galaxies in the Hubble Ultra Deep Field using magphys (da Cunha et al. 2008). ${ }^{23}$ By assuming energy balance between the absorbed-UV radiation from young stars and the reprocessed light from dust, this method predicts dust luminosities based on observed optical/NIR/MIR photometry. The IR luminosities predicted with these two methods are then transformed into $L_{\mathrm{CO}}^{\prime}$ via the empirical relation between $\mathrm{CO}(1-0)$ and dust luminosity (Daddi et al. 2008; Genzel et al. 2010; Carilli \& Walter 2013). Finally, da Cunha et al. (2013b) derive fluxes of higher- $J$ transitions by assuming two extreme cases, a lowCO-excitation scenario, with a Milky-Way-like CO ladder, and a high-CO-excitation scenario, with a $\mathrm{CO}$ ladder resembling the center of the starbursting galaxy M82. The CO line energy distributions in these two cases are taken from Weiß et al. (2007).

The observed distribution resulting from our blind $\mathrm{CO}$ search suggests an excess of sources with respect to the predictions, in particular if we consider all the 11 high-quality line candidates. We stress that a fraction of them is likely not real. We discuss the implications of our observations regarding the CO luminosity functions and the molecular gas density of the universe as a function of redshift in W14.

\footnotetext{
23 The predictions are done for the Hubble Ultra Deep Field (UDF) which is not the HDF-N studied here. We chose the UDF comparison as the available multi-wavelength data needed for the predictions is deeper and more complete for the UDF. We do not expect significant differences in galaxy properties between the HDF-N studied here and the UDF.
}

Table 4

Catalog of the (Four Positive, One Negative) Sources Detected within the Primary Beam of Our Observations in the Collapsed $3 \mathrm{~mm}$ Continuum

\begin{tabular}{lcccc}
\hline \hline ID & Alt. Name & $\begin{array}{c}\text { R.A. } \\
(J 2000.0)\end{array}$ & $\begin{array}{c}\text { Decl. } \\
(\mathrm{J} 2000.0)\end{array}$ & $\begin{array}{c}\text { Flux } \\
(\mu \text { Jy }) \\
(1)\end{array}$ \\
$(2)$ & $(3)$ & $(4)$ & $56 \pm 10$ \\
\hline C1 & HDF 850.1 & $12: 36: 51.94$ & $+62: 12: 26.0$ & $58 \pm 11$ \\
C2 & & $12: 36: 48.50$ & $+62: 12: 32.4$ & $36 \pm 15$ \\
C3 & & $12: 36: 47.57$ & $+62: 12: 35.2$ & $46 \pm 12$ \\
C4 & & $12: 36: 47.99$ & $+62: 12: 17.5$ & $39 \pm 12$ \\
C5 & & $12: 36: 50.63$ & $+62: 12: 42.4$ & $-47 \pm 12$ \\
\hline
\end{tabular}

Notes. Fluxes and uncertainties are corrected for primary beam attenuation.

\section{THE CONTINUUM IMAGE}

By collapsing our molecular line scan in frequency space we obtained a $3 \mathrm{~mm}$ continuum map with an rms of $8.6 \mu \mathrm{Jy}_{\text {beam }}{ }^{-1}$, making this by far the deepest map at this frequency to date (see Figure 18). The image has been cleaned down to the $2 \sigma$ level. We report four sources brighter than $>3 \sigma$, all consistent with being spatially unresolved. Coordinates and fluxes are reported in Table 4. The brightest detection (ID.C1) is associated with the SMG HDF 850.1, and it is spatially consistent with the lines ID. 08 and ID. 17 in Table 2. ID.C4 is marginally consistent with the line candidate ID.01 (the offset is 2 ".7). For the remaining two continuum sources (ID.C2 and ID.C3), we do not report any 
significant line association in our $3 \mathrm{~mm}$ scan. They are likely spurious, as suggested by the detection of an equally significant ( $\gtrsim 3 \sigma)$ negative peak (dubbed ID.C5).

The deep continuum map allows us to constrain the observed SED of known galaxies at an observed wavelength of $3 \mathrm{~mm}$. We stack the $3 \mathrm{~mm}$ continuum image at the coordinates of optical/ NIR/MIR-selected galaxies. The stacks are performed both in the $(\mathrm{U}, \mathrm{V})$ and sky plane, focusing on the following samples: (1) sources with spectroscopic redshift from optical/NIR studies (Bundy et al. 2009, plus additional eight unpublished slit redshifts and 23 grism redshifts, see W14), split into galaxies above and below $z=1$ (in order to have similar sub-sample sizes at low and high $z$ ). As discussed in W14, these samples are highly complete up to $z \sim 3$ for $m_{\mathrm{H}}<24$ mag (roughly corresponding to $M_{*}=7 \times 10^{9} M_{\odot}$ at $z=2-3$ ); (2) optically selected galaxies from the compilation in Fernández-Soto et al. (1999), split again according to their photometric redshift (below or beyond $z_{\text {phot }}=1$ ); (3) IRAC $3.6 \mu \mathrm{m}$ detected sources from the Spitzer Legacy Project on GOODS (PI: M. Dickinson); (4) a sub-sample of the IRAC list, selected by requiring $3.6 \mu \mathrm{m}$ fluxes brighter than $3.5 \mu \mathrm{Jy}$; (5) MIPS-selected galaxies, i.e., sources with MIPS $24 \mu \mathrm{m}$ fluxes brighter than $20 \mu \mathrm{Jy}$. From Figure 18, it is apparent that there is substantial overlap among some galaxy samples, especially if only the brightest sources are considered. We note that each sample consists of $<100$ sources, i.e., the sample sizes are all significantly lower than the number of the resolution elements in the map.

The stacking technique is similar with the one presented in Decarli et al. (2014), and works as follows: we crop the input catalogs to consider only sources within the primary beam of our observations. As for the reference frequency (needed to compute the size of the primary beam), we refer to the central frequency of the scan $(97.25 \mathrm{GHz})$, which implies a fiducial diameter of the primary beam of $48^{\prime \prime} 6$. For each source, we compute its offset with respect to the pointing center. These offset values are used to correct for primary beam attenuation and to compute weights, so that a source lying at the primary beam radius is scaled up by a factor two in flux and is assigned a weight of 0.25 (i.e., the squared value of the primary beam attenuation). In the (U,V) stacking, we shift the sources into the pointing center by applying phase shifts, and then we stack amplitudes. In the image plane stacking, we cut out postage stamps of the sources from the continuum map, realign and combine. In order to quantify the contribution of bright outliers we apply a jack-knife analysis: for each sample of $N$ galaxies, we perform $N$ stacks of $N-1$ sources, by removing a different source each time. Finally, we quantify the uncertainties by stacking on random coordinates within the primary beam. The stacking uncertainties are estimated as the standard deviations of the stacked fluxes over 50 realizations.

No detection is reported in the stacked $3 \mathrm{~mm}$ continuum image of any of the samples considered in our stacking analysis. Table 5 summarizes the results of this stacking experiment. We achieve $1 \sigma$ stack sensitivities of a few $\mu \mathrm{Jy}$ in all samples. We compute the associated limits on the dust continuum emission by assuming a fiducial redshift $z=1$ for all the samples, and shifting the M 51 and M 82 templates by Silva et al. (1998) in order to match the $3 \sigma$ limits from the stacks at the central frequency of our scan $(97.25 \mathrm{GHz}$, our results are practically unchanged for different assumptions of the fiducial redshift, for any $z \gtrsim 1$ ). The M 51 and M 82 templates are adopted to represent a typical spiral galaxy and a starbursting galaxy, respectively.
Table 5

Limits on the $3 \mathrm{~mm}$ Continuum from the Stacking Analysis

\begin{tabular}{|c|c|c|c|c|c|c|c|c|}
\hline \multirow{3}{*}{$\begin{array}{r}\text { Sample } \\
\text { (1) }\end{array}$} & \multirow{3}{*}{$\begin{array}{l}N \\
\\
(2)\end{array}$} & \multirow{3}{*}{$\begin{array}{c}\mathrm{rms} \\
(\mu \mathrm{Jy}) \\
(3)\end{array}$} & \multirow{3}{*}{$\begin{array}{l}z_{\min } \\
\text { (4) }\end{array}$} & \multirow{3}{*}{$\begin{array}{c}z_{\max } \\
(5)\end{array}$} & \multicolumn{2}{|c|}{$\log L_{\mathrm{IR}}$} & \multicolumn{2}{|c|}{ SFR } \\
\hline & & & & & \multicolumn{2}{|c|}{$\left(L_{\odot}\right)$} & \multicolumn{2}{|c|}{$\begin{array}{c}\mathrm{M} 51 \quad \mathrm{M} 82 \\
\left(M_{\odot} \mathrm{yr}^{-1}\right)\end{array}$} \\
\hline & & & & & (6) & (7) & (8) & (9) \\
\hline$z_{\text {spec }}<1$ & 20 & 3.9 & 0 & 1 & 11.04 & 11.92 & 14 & 110 \\
\hline$z_{\text {spec }}>1$ & 17 & 4.0 & 1 & 3.23 & 11.05 & 11.93 & 15 & 110 \\
\hline F99, $z<1$ & 48 & 3.0 & 0 & 1 & 10.93 & 11.81 & 11 & 80 \\
\hline $\mathrm{F} 99, z>1$ & 88 & 2.5 & 1 & 5.64 & 10.85 & 11.73 & 9 & 70 \\
\hline IRAC & 43 & 2.9 & 0 & $\sim 4$ & 10.91 & 11.79 & 11 & 80 \\
\hline IRAC bright & 12 & 5.0 & 0 & $\sim 4$ & 11.15 & 12.03 & 18 & 140 \\
\hline MIPS bright & 9 & 6.3 & 0 & $\sim 4$ & 11.25 & 12.13 & 23 & 170 \\
\hline
\end{tabular}

Notes. The luminosity limits are computed by assuming a fiducial $z=1$, the M51/M82 templates by Silva et al. (1998), and a $3 \sigma$ detection. Associated star formation rates are derived assuming $\operatorname{SFR} /\left[M_{\odot} \mathrm{yr}^{-1}\right]=1.3 \times L_{\mathrm{IR}} /\left[10^{10} L_{\odot}\right]$ (Genzel et al. 2010).

Our $3 \mathrm{~mm}$ continuum observations sample the RayleighJeans tail of the dust emission. Therefore, for a given $3 \mathrm{~mm}$ continuum flux, the inferred luminosity (as reported in Table 5) is a factor $\sim 7$ brighter using the M 82 template compared to the M 51 case, due to the higher dust temperature. Our $3 \sigma$ IR luminosity limits are in the range $(5-20) \times 10^{10} L_{\odot}$ for M51like galaxies, and (4-13) $\times 10^{11} L_{\odot}$ for M82-like dust emission. We convert these limits into constraints on the associated SFR by assuming SFR $/\left[M_{\odot} \mathrm{yr}^{-1}\right]=1.3 \times L_{\mathrm{IR}} /\left[10^{10} L_{\odot}\right]($ Genzel et al. 2010). The resulting SFR $3 \sigma$ limits in our galaxy samples range between 7 and $23 M_{\odot} \mathrm{yr}^{-1}$ in the spiral galaxy case and between 50 and $170 M_{\odot} \mathrm{yr}^{-1}$ in the starburst case. We compute the contribution of each sample to the cosmic SFR densities (SFRDs; see Figure 19) in the case of an M51-like dust template. In order to compute the sampled cosmic volume in each galaxy set, we proceed as follows: (1) for galaxy samples with spectroscopic or photometric $z<1$, we adopt $z_{\min }=0$, $z_{\max }=1$; (2) for the $z>1$ samples, the upper end of the cosmic volume is fixed by the highest- $z$ galaxy in the sample; (3) for galaxy samples without available redshift information, we assume a broad redshift window ranging between $z_{\min }=0$ and $z_{\max }=3-5$. Following da Cunha et al. (2013a), we correct for the cosmic microwave background (CMB), which acts as an observing background and sets a minimum dust temperature. The net effect is that only a fraction of the intrinsic flux of the Rayleigh-Jeans tail of the dust SED is observed. At $3 \mathrm{~mm}$, the correction is $\lesssim 5 \%$ at $z<1$, and up to $\sim 50 \%$ at $z=3$ for a relatively low dust temperature (as in the M51 case) (da Cunha et al. 2013a).

We find that at $z<1$, the limits on the cosmic SFRD set by $3 \mathrm{~mm}$ observations are not stringent, once compared with the observed SFRD evolution (we here compare with the radio/ IR-based results by Reddy et al. 2008; Rujopakarn et al. 2010; Karim et al. 2011). This is partially due to the fact that the cosmic volume probed in our observations at $z<1$ is small. The $z>1$ spectroscopic and photometric samples account for up to about 50 and $65 \%$ of the total cosmic SFRD, respectively. We estimate a similar contribution for IRAC-detected galaxies (although with larger uncertainties, due to the assumptions on the sampled cosmic volume). Half of this contribution comes from IRAC-bright and MIPS-bright sources. If we adopt an M82-like dust template, our SFRD limits are shifted up by a factor $\sim 8$. Our results suggest that, at $1<z \lesssim 3$, optical/ MIR bright galaxies with $M_{*} \gtrsim 10^{10} M_{\odot}$ contribute only to 


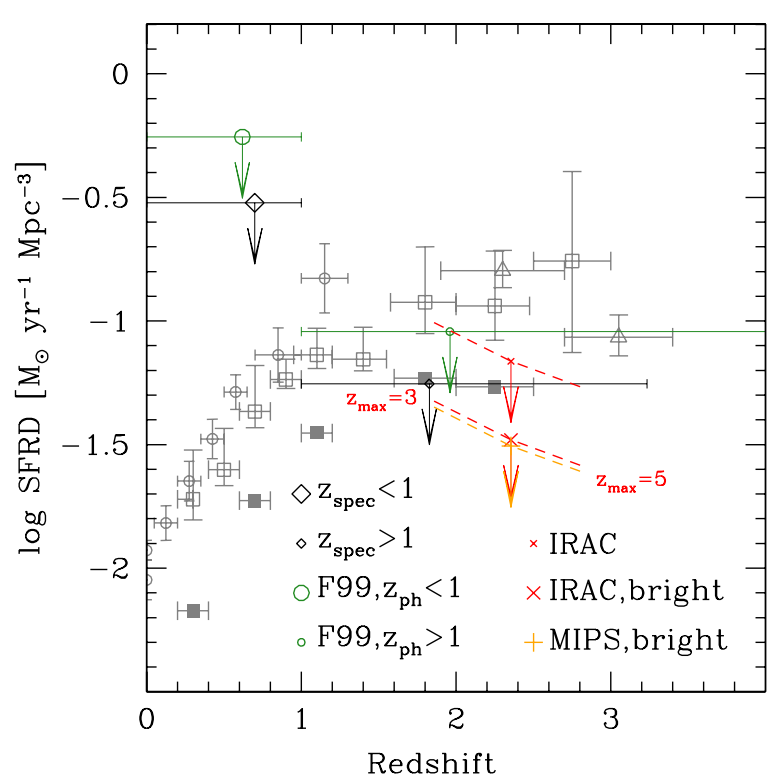

Figure 19. Constraints on the cosmic star formation rate density resulting from our stacking analysis of the $3 \mathrm{~mm}$ continuum image presented in this work, assuming an M51-like dust template to convert observed $3 \mathrm{~mm}$ fluxes into IR luminosities. Symbols are the same as in Figure 18. The $3 \sigma$ limits quoted in Table 5 have been divided by the comoving volume covered by each galaxy sample, and scaled up to take into account the effects of CMB, following da Cunha et al. (2013a). For galaxies with redshift information, the position of the points along the $x$-axis is set by the average redshift of each sample. As for the samples of galaxies without redshift information, we assume $z_{\min }=0$ and $z_{\max }=4$, and place our constraint at the volume-averaged redshift. Dashed lines show how our limits would change if $z_{\max }$ changed from $z=3$ to $z=5$. For a comparison, we plot IR/radio-based SFR density estimates from the literature: empty gray squares from Karim et al. (2011), circles from Rujopakarn et al. (2010) and triangles from Reddy et al. (2008), homogenized to a Chabrier (2003) initial mass function. Filled squares show the results from Karim et al. (2011), once we consider only galaxies with $M_{*}>10^{10} M_{\odot}$. We note that our constraints on SFRD would shift up-ward if a template with higher $T_{\text {dust }}$ was adopted. For example, using M82 as a template would imply $\sim 8 \times$ higher SFRDs for the same $3 \mathrm{~mm}$ fluxes.

(A color version of this figure is available in the online journal.)

a minor fraction of the SFRD at those redshifts, unless their dust SED is better described by starburst template (M82-like, with $T_{\text {dust }} \approx 40 \mathrm{~K}$ ) than by local spiral galaxies (M51-like, with $T_{\text {dust }} \approx 15 \mathrm{~K}$ ). Karim et al. (2011) studied the cosmic SFRD as a function of both redshift and stellar masses. Their results show that star-forming galaxies with $M_{*}>10^{10} M_{\odot}$ contribute to about $50 \%$ of the total SFRD at any redshift between $z=0$ and $z \sim 2.5$, in broad agreement with our findings.

\section{CONCLUSIONS}

We have obtained the first frequency scan of the $3 \mathrm{~mm}$ window (79.7-114.8 GHz) in the HDF-N using the PdBI. This molecular line scan constitutes the first blind survey of $\mathrm{CO}$ emission in "normal" galaxies (i.e., non-SMGs) at high $z$. Our main observational results can be summarized as follows:

1. We achieved approximately uniform sensitivity ( $\sim 0.3 \mathrm{mJy} \mathrm{beam}^{-1}$ per $90 \mathrm{~km} \mathrm{~s}^{-1}$ channel) over the entire scan, which allows us to probe the $\mathrm{CO}$ line luminosities down to (4-8) $\times 10^{9} \mathrm{~K} \mathrm{~km} \mathrm{~s}^{-1} \mathrm{pc}^{2}$ at any $z>1$.

2. Using different search algorithms we have identified 17 positive line candidates (and 4 negative ones). Typical fluxes are in the range $0.16-0.56 \mathrm{Jy} \mathrm{km} \mathrm{s}^{-1}$, while line widths are between 110 and $500 \mathrm{~km} \mathrm{~s}^{-1}$. We estimate that our completeness is $\sim 66 \%$ for the faintest targets, and close to $100 \%$ for the brightest. The rate of false positives is about 2 in the whole scan.

3. Two line detections (ID.08 and ID.17) are spatially consistent with the SMG HDF 850.1 at $z=5.183$ (Walter et al. 2012). In the Appendix of this paper we present new $2 \mathrm{~mm}$ observations encompassing the $\mathrm{CO}(7-6)$ and $\left[\mathrm{C}_{\mathrm{I}}\right]_{2-1}$ lines of this source.

4. No second line is reported at the position of any of the other line candidates, suggesting that, if real, the candidate lines belong to galaxies at intermediate to low redshift $(z \lesssim 3)$.

5. Besides HDF 850.1, two line candidates (ID.03 and ID.19) are considered "secure," being independently confirmed by corollary data. ID.03 is the brightest line in our scan. It is associated with a FIR-bright $B z K$ galaxy. Our follow-up observations with PdBI at $2 \mathrm{~mm}$ and with JVLA in $Q$ band allow us to pin down the redshift of this source $z=1.784$, in excellent agreement with the photometric redshift and its SED. This galaxy lies within the scatter of the "mainsequence" relation of galaxies. For this source, we report a higher $\mathrm{CO}$ excitation than what has been observed in other $B z K$ galaxies to date. The other secure line, ID.19, has also an optical/NIR counterpart, and fulfills the classification of a $B z K$ galaxy. This source has an available (grism) redshift, consistent with the one inferred from our $\mathrm{CO}$ detection, at $z=2.048$. Among the remaining line candidates, nine (ID.01, 02, 05, 10, 11, 12, 15, 18, 20) are considered "highquality."

6. We present a detailed study of optical/NIR counterparts of our line candidates. We found one or more optical/NIR galaxies that are located within the positional uncertainties of seven line candidates (ID.02, 03, 04, 12, 19, 20, 21). We model the optical/NIR/MIR photometry of these tentative counterparts assuming various $\mathrm{CO}$ identifications of the line candidates, and we use the goodness of the fit as a criterion to discriminate among different redshift scenarios, when no corollary spectroscopic information is available. We note that some high-quality $\mathrm{CO}$ line candidates in our survey (e.g., ID.18) lack of any counterpart at other wavelengths, despite the exquisite depth of the available optical/NIR/ MIR data.

7. We report a possible excess in the fluxes of our line candidates with respect to the predictions based on the evolution of galaxy luminosity functions (Sargent et al. 2012, 2013) as well as extrapolation from optical/NIR emissions (da Cunha et al. 2013b). However, we caution that this comparison includes all the "secure" and "highquality" line candidates, although some of them may not be real (as they still need to be confirmed through followup observations of higher- $J$ lines). In an accompanying paper (W14), we use our measurements to constrain the $\mathrm{CO}$ luminosity function and the evolution of the $\mathrm{H}_{2}$ mass density as a function of lookback time.

8. By collapsing our data cube along the frequency axis, we obtain the deepest $3 \mathrm{~mm}$ continuum image available to date. We clearly detect HDF 850.1, and three additional continuum candidates at $\sim 3 \sigma$ level. Only one of these fainter sources show a tentative association with a line candidate (ID.01). Neither HDF 850.1 nor any of the three other continuum candidates is detected in any optical or IR survey.

9. Using various samples of galaxies selected based on their optical or MIR emission, we place sensitive constraints 

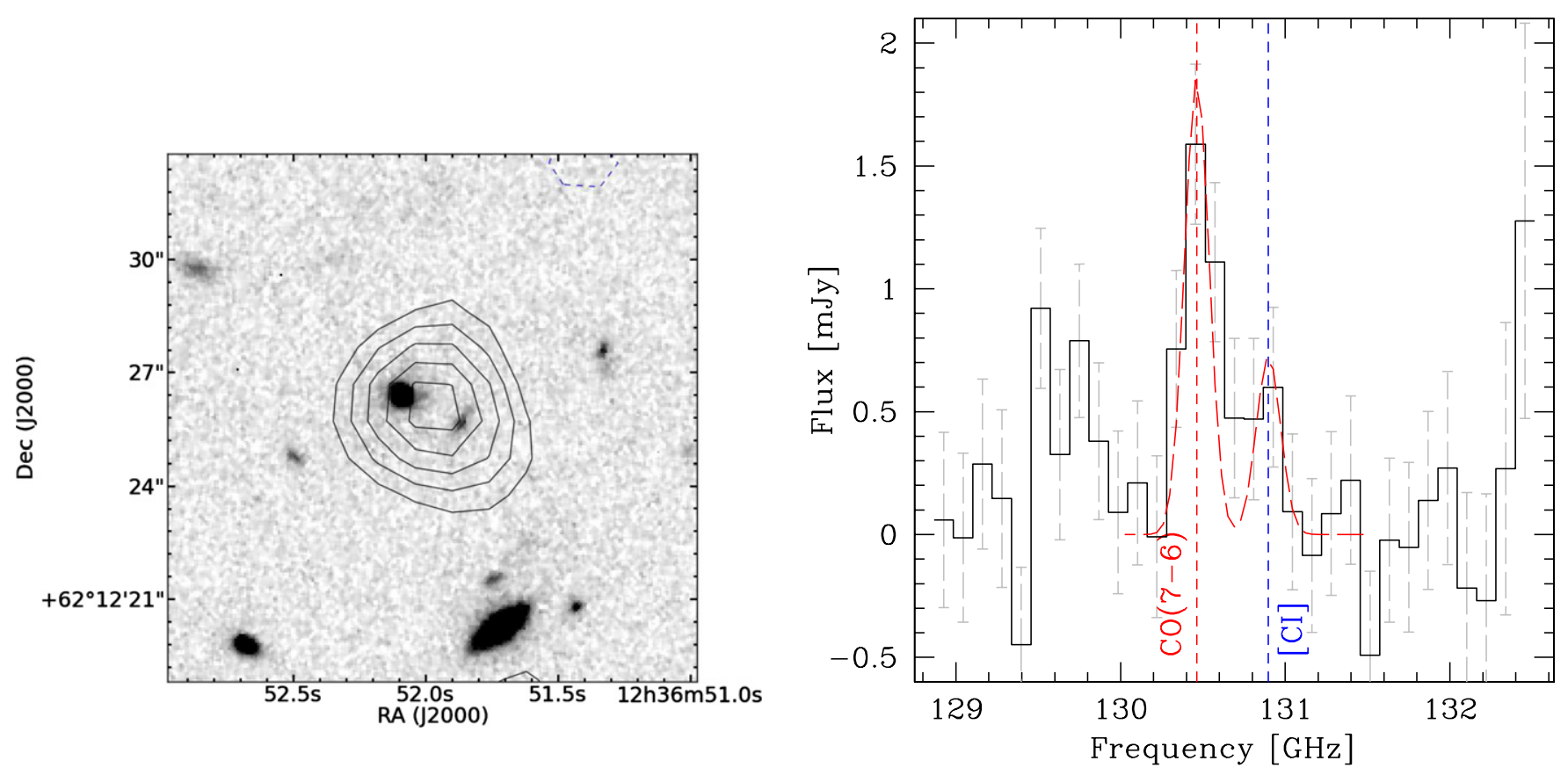

Figure 20. Line map and spectrum of the $\mathrm{CO}(7-6)+[\mathrm{C} \mathrm{I}]$ system of HDF 850.1 at $z=5.183$. Left: the line map is integrated over $1530 \mathrm{~km} \mathrm{~s}{ }^{-1}$, encompassing both $\mathrm{CO}(7-6)$ and $\left[\mathrm{C}_{\mathrm{I}}\right]$. The gray scale is the HST/WFC3 F160W image of the field from the CANDELS survey (Grogin et al. 2011; Koekemoer et al. 2011). Black solid/blue dashed contours show the $2,3,4, \ldots \sigma$ positive/negative isophotes in the $\mathrm{CO}$ map. The box is $14^{\prime \prime} \times 14^{\prime \prime}$ wide. Right: the observed $\mathrm{CO}(7-6)+[\mathrm{C} \mathrm{I}]$ line profile is plotted as a solid histogram, rebinned to $270 \mathrm{~km} \mathrm{~s}^{-1}$ channel width. We fit the line profiles with two Gaussians (red, dashed lines) fixed in redshift and width at the parameters obtained from the $\mathrm{CO}(5-4)$ and $\mathrm{CO}(6-5)$ transitions (line candidates ID.08 and ID.17 in this work; see Walter et al. 2012).

(A color version of this figure is available in the online journal.)

on the dust continuum emission of galaxies at $3 \mathrm{~mm}$. Our results suggest that, at $1 \lesssim z \lesssim 3$, optical and MIR bright galaxies with $M_{*} \gtrsim 10^{10} M_{\odot}$ contribute only to a fraction $(<50 \%)$ of the SFRD at those redshifts, unless their dust SED is better described by starburst template (M82-like, with $T_{\text {dust }} \approx 40 \mathrm{~K}$ ) than by local spiral galaxies (M51-like, with $T_{\text {dust }} \approx 15 \mathrm{~K}$ ).

This study proves the feasibility of sensitive blind molecular line searches in distant galaxies. Parallel endeavors in the radio window (e.g., using JVLA to target low-J CO transitions of high- $z$ galaxies) and at $\sim 1 \mathrm{~mm}$ wavelengths (e.g., in the near future capitalizing on the upgraded capabilities of NOEMA/ PdBI and ALMA), will provide us with a new, complete description of the molecular gas properties of star-forming galaxies through cosmic times.

We thank the referee, S. Serjeant, for his useful comments which improved the quality of our paper. This work is based on observations carried out with the IRAM Plateau de Bure Interferometer. IRAM is supported by INSU/CNRS (France), MPG (Germany) and IGN (Spain). This research made use of Astropy, a community-developed core Python package for Astronomy (The Astropy Collaboration 2013). Support for RD was provided by the DFG priority program 1573 "The physics of the interstellar medium.” F.W., D.R. and E.d.C. acknowledge the Aspen Center for Physics where parts of this manuscript were written.

\section{APPENDIX}

\section{2 mm OBSERVATIONS OF HDF 850.1}

We here present PdBI observations at $2 \mathrm{~mm}$ wavelength of the $\mathrm{CO}(7-6)$ and $\left[\mathrm{C}_{\mathrm{I}}\right]_{2-1}$ transitions (rest-frame frequencies:
806.652 GHz and 809.342 GHz, respectively) in HDF 850.1 that have been obtained as a follow-up of the molecular line scan discussed in this paper. These new observations were carried out in four tracks between 2011 December 3 and 11. The receiver frequency was tuned to $130.680 \mathrm{GHz}$, thus encompassing $\mathrm{CO}(7-6)$ and $\left[\mathrm{CI}_{2}\right]_{2-1}$ at $z=5.183$. At this frequency, the primary beam of PdBI antennas is 36 ". 2 (FWHM). The pointing center of the observations was at R.A. = 12:36:51.90, decl. = +62:12:25.7 (J2000.0). The array was in compact configuration (five or six antennas), with baselines ranging between $17.7 \mathrm{~m}$ and $97.6 \mathrm{~m}$. Data were reduced and analyzed using GILDAS, as described in Section 2. We binned the cube into $90 \mathrm{~km} \mathrm{~s}^{-1}$ channels. The resolution element is $4^{\prime \prime} 0 \times 3^{\prime \prime} .8$. The final cube has 7018 visibilities, corresponding to $5.85 \mathrm{hr}$ on source (sixantenna equivalent). The noise per $90 \mathrm{~km} \mathrm{~s}^{-1}$ channel is 0.60 mJy beam $^{-1}$.

The resulting spectrum of the $\mathrm{CO}(7-6)$ and $[\mathrm{C}]_{2-1}$ transitions extracted on the position of HDF 850.1 is presented in Figure 20. $\mathrm{CO}(7-6)$ is detected at $6 \sigma$ level, and there is a $2 \sigma$ peak at the frequency of [CI]. We fit these two peaks with two Gaussians with fixed peak frequency and width, as derived from the lower- $J$ CO transitions $\left(z=5.183\right.$, FWHM $_{\mathrm{CO}}=$ $400 \mathrm{~km} \mathrm{~s}^{-1}$ ). We obtain $S_{\mathrm{CO}(7-6)}=1.86 \pm 0.30 \mathrm{mJy}$ and $S_{[\mathrm{CI}]}=0.73 \pm 0.30 \mathrm{mJy}$, yielding total line fluxes of $0.35 \pm 0.05$ $\mathrm{Jy} \mathrm{km} \mathrm{s}{ }^{-1}$ and $0.14 \pm 0.05 \mathrm{Jy} \mathrm{km} \mathrm{s}^{-1}$, and line luminosities $L=$ $(2.4 \pm 0.4) \times 10^{8} L_{\odot}, L^{\prime}=(1.4 \pm 0.2) \times 10^{10} \mathrm{~K} \mathrm{~km} \mathrm{~s}^{-1} \mathrm{pc}^{2}$, and $L=(5.7 \pm 2.3) \times 10^{7} L_{\odot}, L^{\prime}=(1.5 \pm 0.6) \times 10^{10} \mathrm{~K} \mathrm{~km} \mathrm{~s}^{-1} \mathrm{pc}^{2}$, for $\mathrm{CO}(7-6)$ and $[\mathrm{CI}]_{2-1}$ respectively. Walter et al. (2012) based on the $\mathrm{CO}(2-1), \mathrm{CO}(5-4)$ and $\mathrm{CO}(6-5)$ lines infer a $\mathrm{CO}(1-0)$ line luminosity of $L^{\prime}=4.3 \times 10^{10} \mathrm{~K} \mathrm{~km} \mathrm{~s}^{-1} \mathrm{pc}^{2}$. This implies a $L^{\prime}(\mathrm{CO}(7-6)) / L^{\prime}(\mathrm{CO}(1-0))=0.33$, which, within the uncertainties, is thus is broadly consistent with other highredshift FIR-bright galaxies (Carilli \& Walter 2013; Riechers et al. 2013). 


\section{REFERENCES}

Aravena, M., Carilli, C., Daddi, E., et al. 2010, ApJ, 718, 177

The Astropy Collaboration, Robitaille, T. P., Tollerud, E. J., Greenfield, P., et al. 2013, A\&A, 558, A33

Barger, A. J., Cowie, L. L., \& Wang, W.-H. 2008, ApJ, 689, 687

Bothwell, M. S., Smail, I., Chapman, S. C., et al. 2013, MNRAS, 429, 3047

Bundy, K., Ellis, R. S., \& Conselice, C. J. 2009, yCat, 7246, 0

Carilli, C. L., Hodge, J., Walter, F., et al. 2011, ApJL, 739, L33

Carilli, C. L., \& Walter, F. 2013, ARA\&A, 51, 105

Casey, C. M., Chapman, S. C., Neri, R., et al. 2011, MNRAS, 415, 2723

Chabrier, G. 2003, PASP, 115, 763

da Cunha, E., Charlot, S., \& Elbaz, D. 2008, MNRAS, 388, 1595

da Cunha, E., Groves, B., Walter, F., et al. 2013a, ApJ, 766, 13

da Cunha, E., Walter, F., Decarli, R., et al. 2013b, ApJ, 765, 9

Daddi, E., Bournaud, F., Walter, F., et al. 2010, ApJ, 713, 686

Daddi, E., Cimatti, A., Renzini, A., et al. 2004, ApJ, 617, 746

Daddi, E., Dannerbauer, H., Elbaz, D., et al. 2008, ApJL, 673, L21

Daddi, E., Dannerbauer, H., Stern, D., et al. 2009, ApJ, 694, 1517

Dannerbauer, H., Daddi, E., Riechers, D. A., et al. 2009, ApJL, 698, L178

Decarli, R., Smail, I., Walter, F., et al. 2014, ApJ, 780, 115

Dickinson, M., Papovich, C., Ferguson, H. C., \& Budavári, T. 2003, ApJ, 587,25
Elbaz, D., Dickinson, M., Hwang, H. S., et al. 2011, A\&A, 533, 119

Fernández-Soto, A., Lanzetta, K. M., \& Yahil, A. 1999, ApJ, 513, 34

Genzel, R., Tacconi, L. J., Gracia-Carpio, J., et al. 2010, MNRAS, 407, 2091

Grogin, N. A., Kocevski, D. D., Faber, S. M., et al. 2011, ApJS, 197, 35

Hughes, D. H., Serjeant, S., Dunlop, J., et al. 1998, Natur, 394, 241

Karim, A., Schinnerer, E., Martínez-Sansigre, A., et al. 2011, ApJ, 730, 61

Koekemoer, A. M., Faber, S. M., Ferguson, H. C., et al. 2011, ApJS, 197, 36

Reddy, N. A., Steidel, C. C., Pettini, M., et al. 2008, ApJS, 175, 48

Riechers, D. A., Bradford, C. M., Clements, D. L., et al. 2013, Natur, 496, 329

Rosolowski, E., \& Leroy, A. 2006, PASP, 118, 590

Rujopakarn, W., Eisenstein, D. J., Rieke, G. H., et al. 2010, ApJ, 718, 1171

Sargent, M. T., Béthermin, M., Daddi, E., \& Elbaz, D. 2012, ApJL, 747, L31

Sargent, M. T., Daddi, E., Béthermin, M., et al. 2013, ApJ, submitted (arXiv:1303.4392)

Silva, L., Granato, G. L., Bressan, A., \& Danese, L. 1998, ApJ, 509, 103

Walter, F., Decarli, R., Carilli, C., et al. 2012, Natur, 486, 233

Walter, F., Decarli, R., \& Sargent, M. 2014, ApJ, 782, 79

Weiß, A., De Breuck, C., Marrone, D. P., et al. 2013, ApJ, 767, 88

Weiß, A., Downes, D., Walter, F., \& Henkel, C. 2007, in ASP Conf. Ser. 375, From Z-Machines to ALMA: (Sub)Millimeter Spectroscopy of Galaxies, ed. A. J. Baker, J. Glenn, A. I. Harris, J. G. Mangum, \& M. S. Yun (San Francisco, CA: ASP), 25

Williams, R. E., Blacker, B., Dickinson, M., et al. 1996, AJ, 112, 1335 